Supporting Information for

\title{
Rational Ligand Design for an Efficient Biomimetic Water Splitting Complex
}

\author{
Penglin $\mathrm{Xu}^{\ddagger}$, Ting Zhou ${ }^{\ddagger}$, Nadia N. Intan, Shaojin Hu, and Xiao Zheng* \\ Hefei National Laboratory for Physical Sciences at the Microscale \& Synergetic Innovation \\ Center of Quantum Information and Quantum Physics, University of Science and Technology of \\ China, Hefei, Anhui 230026, China \\ $\$$ Authors of equal contribution \\ *To whom correspondence should be addressed: xz58@ustc.edu.cn
}

November 24, 2016

This supplemental file includes the following materials:

1. Some remarks on theoretical methods and computational procedures (p. S2-S3).2. Frequency analysis for transition state complexes (TS1 and TS2) associated with the O-O bond formation and oxygen releasing steps for complex 2 (p. S3-S5).

3. Atomic charge and spin analysis for complex 2 (p. S5).

4. O-O bond formation pathway calculated by using the cc-pVTZ basis set (p. S5-S6).

5. Relative energies of various spin states of the $\mathrm{Mn}^{\mathrm{IV}}-\mathrm{Mn}^{\mathrm{IV}}$ core (p. S6).

6. Contour plot of spin density distribution of complex 2 (p. S6-S7).

7. Optimized geometric structures of complexes $\mathbf{1}$ and $\mathbf{2}$ (and some related species) (p. S8-S41).

8. Mulliken atomic charge and spin population data for complex $\mathbf{2}$ (and some related species) (p. S41-S49). 


\section{Some Remarks on Theoretical Methods and Computational Procedures.}

\subsection{Details on density functional theory calculations}

Molecular geometries and electronic structures are obtained by carrying out density functional theory (DFT) calculations with the hybrid B3LYP exchange-correlation functional. ${ }^{1,2}$ The only exception is the reaction pathway for the oxygen releasing step, which is calculated by using the M06-L functional. ${ }^{3-5}$ All the geometric optimizations and frequency analysis are performed by using a mixed basis set (LanL2DZ ${ }^{6}$ for Mn atoms, 6-31G(2df) for oxo-bridge oxygen atoms, and 6-31G(d) for rest of atoms). The electronic ground states of complex 2 and related dimanganese species are affirmed to be in broken symmetry spin states. ${ }^{7,8}$ To calculate the transition states, the QST3 and TS algorithms of the Gaussian09 suite of program is adopted.

Single point energies are calculated with the mixed basis set (LanL2DZ for Mn atoms, and $6-31 G^{9-10}$ for the rests), from which the thermodynamic energies are extracted. An implicit solvent model, the polarizable continuum model $(\mathrm{PCM})^{11}$ is employed to evaluate the solvation free energy of complex 2 and related molecular species.

\subsection{Calculations for $\mathbf{p K a}$ Values.}

All the pKa values are calculated by using the Born-Haber ( $\mathrm{BH})$ thermodynamic cycle, which has been widely adopted in the literature. ${ }^{12-16}$ Below is a schematic diagram of the $\mathrm{BH}$ cycle of the deprotonation reaction $\mathrm{AH}=\mathrm{A}^{-}+\mathrm{H}^{+}$.

$$
\begin{array}{ll}
A H_{\text {gas }} \stackrel{\Delta G_{g a s}}{\longrightarrow} & A_{\text {gas }}^{-}+H_{g a s}^{+} \\
\downarrow \Delta G_{s}(A H) & \downarrow \Delta G_{s}\left(A^{-}\right) \quad \downarrow \Delta G_{s}\left(H^{+}\right) \\
A H_{a q} \stackrel{\Delta G_{a q}}{\longrightarrow} & A_{a q}^{-}+H_{a q}^{+}
\end{array}
$$

In this cycle, $\Delta G_{\text {gas }}$ is calculated in the gas phase, and the solvation free energy of the protonated species $\Delta G_{S}(A H)$ and that of the deprotonated species $\Delta G_{S}\left(A^{-}\right)$are both computed by using an implicit solvent model. The solvation free energy of a proton $\Delta G_{S}\left(H^{+}\right)$takes the experimental value of $-11.45 \mathrm{eV}$, or can be extracted from highlevel first-principles calculations. The deprotonation reaction free energy is obtained as

$$
\Delta G_{a q}=\Delta G_{g a s}+\Delta G_{s}\left(A^{-}\right)+\Delta G_{s}\left(H^{+}\right)-\Delta G_{s}(A H)
$$

The $\mathrm{pKa}$ value is then computed as $\mathrm{pKa}=\frac{\Delta G_{a q}}{(\ln 10) R T}$.

In the literature a conductor-like PCM (C-PCM) solvation model has been used widely, which is believed to result in reasonably accurate pKa values. ${ }^{12,17-18}$ This is 
because most of the errors in $\Delta G_{s}\left(A^{-}\right)$and $\Delta G_{s}(A H)$ cancel each other out. For instance, George et al. ${ }^{16}$ have calculated the pKa values for carboxylic acids using complete basis set and Gaussian-n models combined with C-PCM continuum solvation methods. The resulting pKa values are accurate to less than half a pKa unit.

By using the $\mathrm{BH}$ cycle, we examine the $\mathrm{pKa}$ value associated with the nitrogen atom $\mathrm{N}(\mathrm{g})$ on the 2-bpnp ligand of complex 2 (see Figure 4 in the main text). The pKa is calculated to be 1.5 (7.8) with the C-PCM ${ }^{19}$ (PCM) solvation model. It is thus concluded that the $\mathrm{N}(\mathrm{g})$ atom is largely unsaturated in the water environment, which makes the O$\mathrm{O}$ bond formation reaction feasible.

All the pKa values listed in Figure 7 in the main text are calculated by using the PCM solvation model.

\subsection{Computation of Gibbs Free Energy.}

The changes in Gibbs free energy along the reaction pathways are calculated with the following formulas: ${ }^{20}$

$\Delta G_{a q}^{298}=\Delta G_{g}^{298}+\Delta G_{\text {solv }}^{298}$

$\Delta G_{g}^{298}=E_{0}+\Delta \Delta H_{g}^{298}-T \Delta S_{g}^{298}$

$\Delta \Delta H_{g}^{298}=\Delta \Delta H_{v i b}^{298}+\Delta \Delta H_{\text {rot }}^{298}+\Delta \Delta H_{\text {trans }}^{298}$

$E_{0}=E_{\text {elec }}+Z P E$

Here, $\Delta G_{g}^{298}$ is the free energy change in the gas phase, which contains the energy at absolute zero (including the zero point energy) and the thermal correction $\Delta \Delta H_{g}^{298}$ (including vibrational, translational, and rotational contributions). $\Delta G_{\text {solv }}^{298}$ is the solvation free energy in water environment, which is calculated by using the implicit solvent model.

\section{Frequency Analysis for Transition State Complexes (TS1 and TS2) Associated} with the O-O Bond Formation and Oxygen Releasing Pathways.

(1). Frequency analysis for transition states associated with one water molecule approaching (a) the antiparallel $\mathrm{Mn}^{\mathrm{IV}}$-oxyl radical (TS1) and (b) the parallel $\mathrm{Mn}^{\mathrm{IV}}$-oxyl radical (TS2) species for complex 2. 


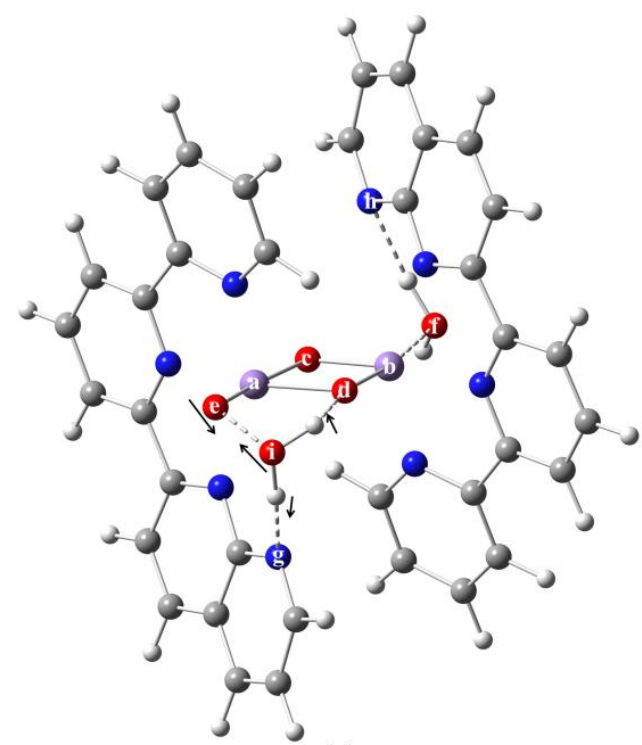

(a)

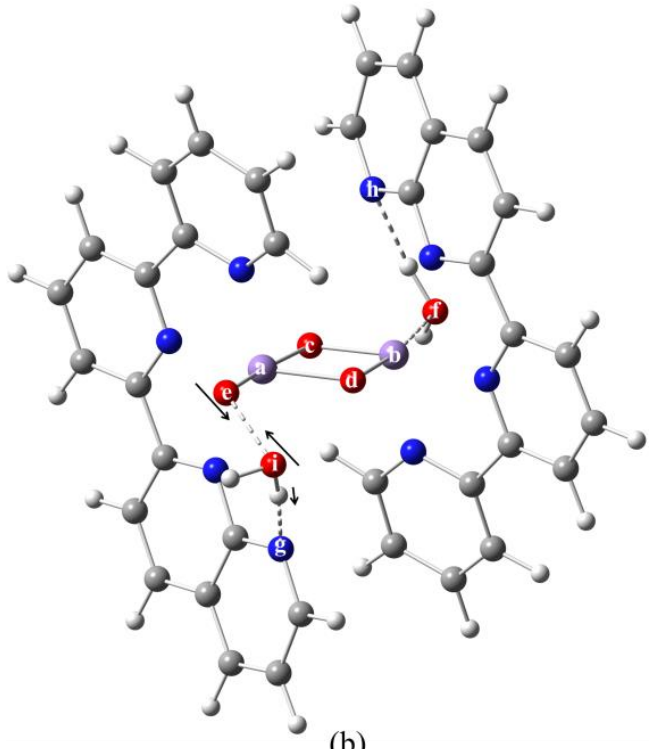

(b)

Figure S1. Vibrational motions of atoms in (a) TS1 and (b) TS2 corresponding to the imaginary frequency. Black arrows indicate the vibration directions.

The imaginary frequencies for TS1 (water molecule approaching the antiparallel $\mathrm{Mn}^{\mathrm{IV}}$ oxyl radical) and TS2 (water molecule approaching the parallel $\mathrm{Mn}^{\mathrm{IV}}$-oxyl radical) are $-666.86 \mathrm{~cm}^{-1}$ and $-953.45 \mathrm{~cm}^{-1}$, respectively. In both cases, the vibration motion correspond to the imaginary-frequency mode that indicates the formation of $\mathrm{O}-\mathrm{O}$ bond.

(2). Frequency analysis for transition states associated with oxygen releasing for (a) complex 2 and (b) complex 1.

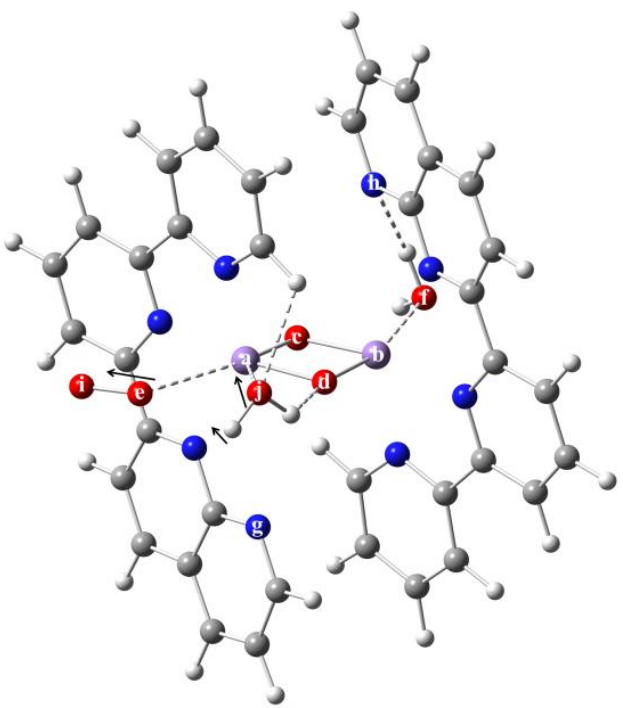

(a)

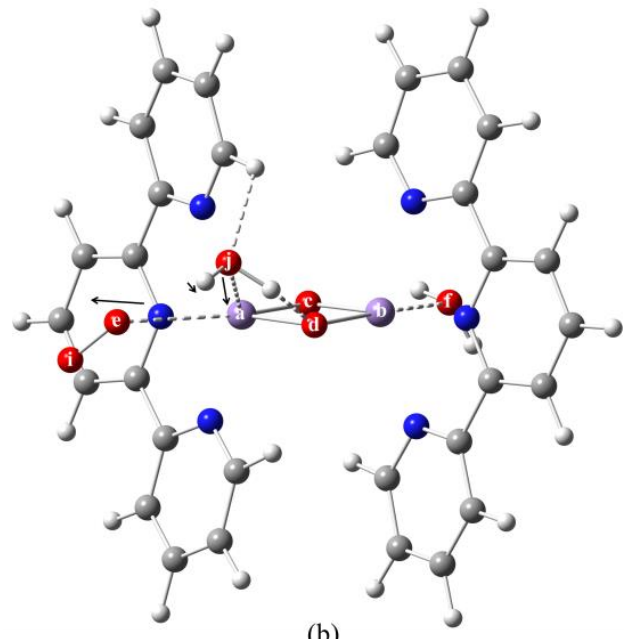

(b) 
Figure S2. Vibrational motions of atoms in oxygen releasing step for (a) complex $\mathbf{2}$ and (b) complex $\mathbf{1}$ corresponding to the imaginary frequency. Black arrows indicate the vibration directions.

The imaginary frequencies associated with the oxygen releasing step for complex 2 and complex 1 are $-68.16 \mathrm{~cm}^{-1}$ and $-49.56 \mathrm{~cm}^{-1}$, respectively. In both cases, the vibration motion correspond to the imaginary-frequency mode that indicates the coordination of $\mathrm{H}_{2} \mathrm{O}$ and the dissociation of oxygen atom.

\section{Atomic Charge and Spin Analysis for Complex 2.}

The optimized structure of complex $\mathbf{2}$ is shown in Figure 3 in the main text. In this work, the atomic charge and spin population on the dimanganese core are mainly studied by using the Mulliken population analysis. The basis set dependence is found rather weak for the dimanganese complexes investigated in this work. The Mulliken atomic charges on $\mathrm{Mn}(\mathrm{III})$ and $\mathrm{Mn}(\mathrm{IV})$ centers are determined to be 1.06 and 1.18, respectively. The atomic charges on the Mn centers deviate significantly from their formal valence charges, indicating that the Mn centers actually accept some electrons from the surrounding ligands through the coordination bonds. We have also carried out a natural population analysis (NPA), which results in atomic charges of 1.57 and 1.67 on $\mathrm{Mn}(\mathrm{III})$ and $\mathrm{Mn}(\mathrm{IV})$ centers, respectively. Apparently, the NPA and Mulliken population data agree consistently with each other.

The Mulliken atomic spin on $\mathrm{Mn}(\mathrm{III})$ and $\mathrm{Mn}(\mathrm{IV})$ centers are 4.00 and -2.83 , and the corresponding NPA atomic spins are 3.87 and -2.63 , respectively. These numbers clearly affirm the antiparallel-spin state of complex $\mathbf{2}$.

\section{O-O Bond Formation Pathway Calculated by Using the cc-pVTZ Basis Set.}

Figure S3 shows the Gibbs free energy profile for the O-O bond formation pathway of complex 2. The calculation results with the cc-pVTZ and the mixed lacvp basis sets are compared in parallel. Apparently, the energy differences due to the different basis set are only $2-3 \mathrm{kcal} / \mathrm{mol}$, and the Mulliken atomic spins agree very well. 


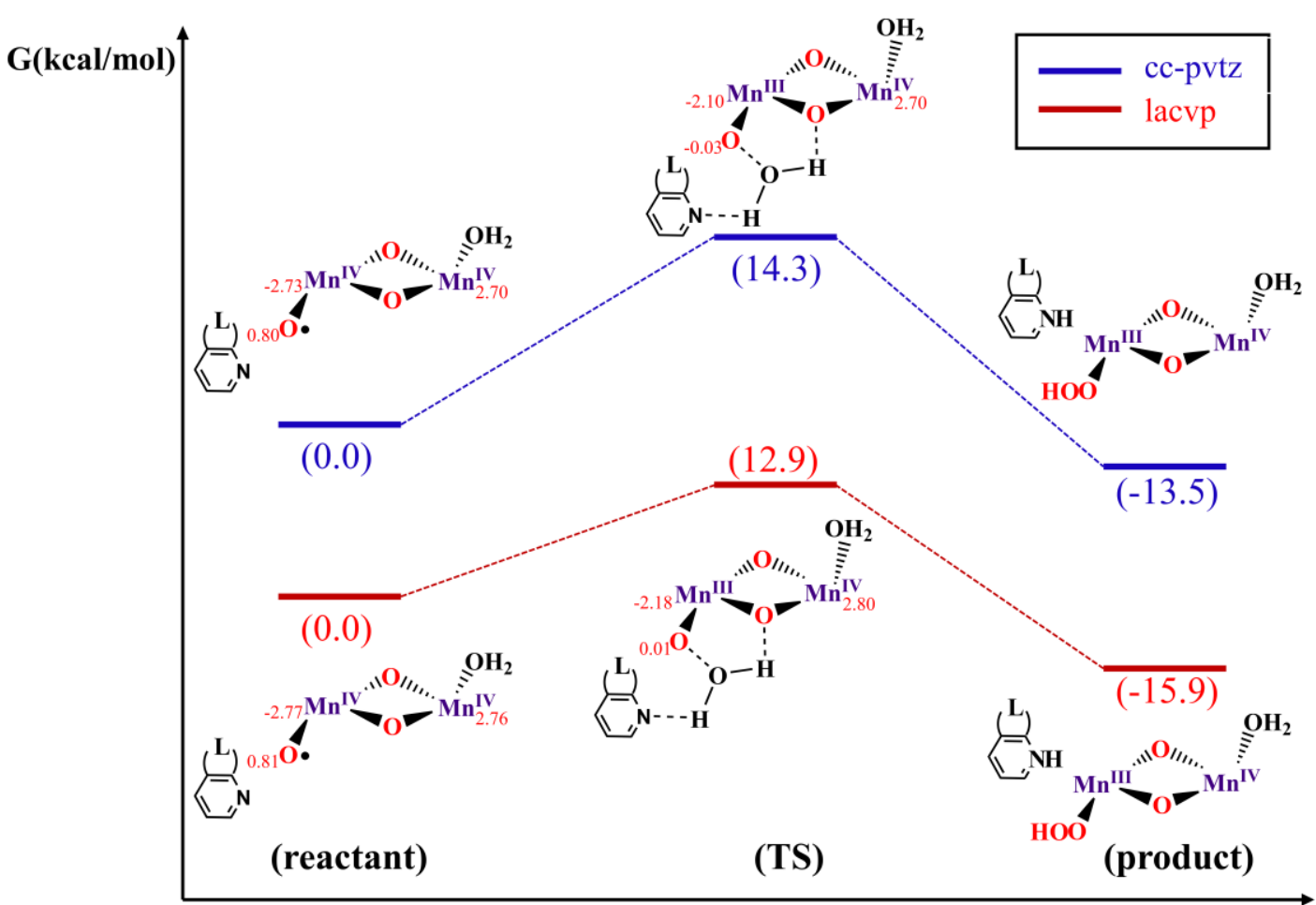

Figure S3. Free energy profile for the O-O bond formation reaction of complex 2. The calculation results with cc-pVTZ and lacvp (Mn with LANL2DZ and the other atoms with 6-31G) basis sets are compared in parallel. The subscripts in red indicate the Mulliken atomic spin population.

\section{Relative Energies of Various Spin States of the $\mathrm{Mn}^{\mathrm{IV}}-\mathrm{Mn}^{\mathrm{IV}}$ Core}

\begin{tabular}{ccccccccc}
\hline & & \multicolumn{3}{c}{ Formal atomic spin } & \multicolumn{3}{c}{ Mulliken atomic spin } & Relative energy \\
\cline { 3 - 7 } No. & multiplicity & Mn1 & Mn2 & O5 & Mn1 & Mn2 & O5 & (kcal/mol) \\
\hline 1 & doublet & -3 & 3 & 1 & -2.75 & 2.74 & 0.80 & 0.0 \\
2 & sextet & 3 & 3 & -1 & 2.80 & 2.78 & -0.80 & 3.51 \\
3 & doublet & 3 & -3 & 1 & 2.65 & -2.76 & 1.12 & 4.15 \\
4 & octet & 3 & 3 & 1 & 2.68 & 2.78 & 1.11 & 10.32 \\
5 & quartet & -1 & 3 & 1 & -1.04 & 2.76 & 1.18 & 15.61 \\
6 & quartet & 1 & 3 & -1 & 1.14 & 2.72 & -1.21 & 17.72 \\
7 & quartet & 3 & 1 & -1 & 2.78 & 0.99 & -0.79 & 20.59 \\
8 & doublet & 3 & -1 & -1 & 2.76 & -1.15 & -0.81 & 21.04 \\
9 & doublet & -1 & 1 & 1 & -1.06 & 1.36 & 1.24 & 33.01 \\
\hline
\end{tabular}

Table S1. Nine spin states of the $\mathrm{Mn}^{\mathrm{IV}}-\mathrm{Mn}^{\mathrm{IV}}$ core of highly oxidized complex 2 .

\section{Contour Plot of Spin Density Distribution of Complex 2.}




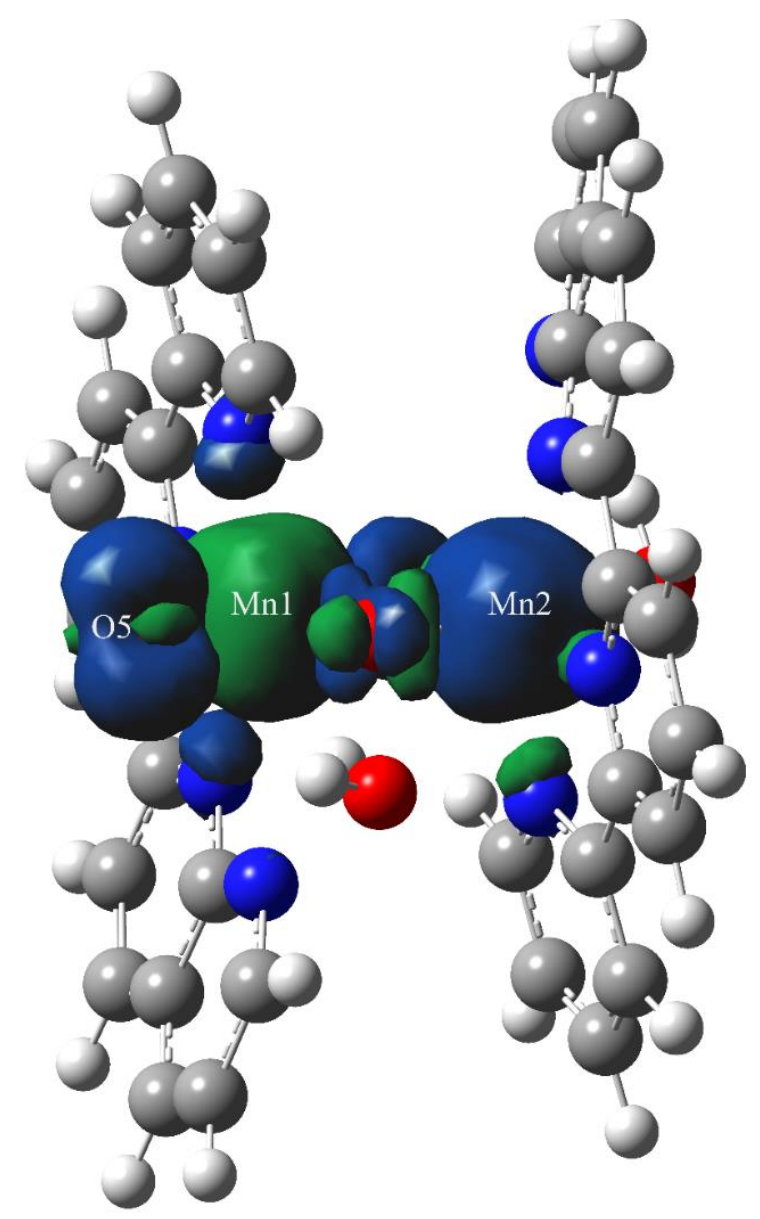

Figure S4. Contour plot of spin density distribution for the antiparallel-spin reactant state of complex 2 . The up- and down-spin densities are indicated by blue and green, respectively. 


\section{Optimized Geometric Structures of Complexes 1 and 2 (and Some Related}

\section{Species)}

Cartesian coordinates of complex 1

Number of atoms in complex 1: 68

\begin{tabular}{|c|c|c|c|}
\hline Mn1 & -1.123340 & 0.001854 & 0.614319 \\
\hline Mn2 & 1. 316363 & 0.003512 & -0.581317 \\
\hline 03 & -0.358677 & 0.005866 & -1.091436 \\
\hline 04 & 0.670716 & 0.000114 & 1. 043605 \\
\hline 05 & -1.467112 & 0.006753 & 2. 710272 \\
\hline 06 & 1. 850233 & 0.008288 & -2.62270 \\
\hline N7 & -1.833132 & -2.165765 & 0.735326 \\
\hline N8 & -3.097478 & -0.005021 & -0.126478 \\
\hline N9 & -1.847471 & 2. 166312 & 0.728347 \\
\hline N10 & 1. 765200 & -1.994528 & -0.624271 \\
\hline N11 & 3. 238427 & 0.000225 & 0.028527 \\
\hline N12 & 1. 768706 & 2. 000882 & -0.613793 \\
\hline $\mathrm{C} 13$ & -3.024627 & -2.393549 & 0.111225 \\
\hline C14 & -3.673340 & -1.193349 & -0.461315 \\
\hline C15 & -4.838325 & -1.222028 & -1.237663 \\
\hline $\mathrm{C} 16$ & -5.413365 & -0.015410 & -1.646434 \\
\hline C17 & -4.844278 & 1. 196503 & -1.24508 \\
\hline $\mathrm{C} 18$ & -3.679275 & 1. 178366 & -0.468415 \\
\hline C19 & -3.037380 & 2. 385134 & 0.098058 \\
\hline C20 & 3. 004211 & -2.336773 & -0.133581 \\
\hline C21 & 3. 816739 & -1.192001 & 0.313989 \\
\hline $\mathrm{C} 22$ & 5. 068810 & -1.222329 & 0.938158 \\
\hline $\mathrm{C} 23$ & 5. 688877 & -0.005145 & 1. 254479 \\
\hline C24 & 5. 070813 & 1. 214769 & 0.944832 \\
\hline C25 & 3. 818710 & 1. 189916 & 0.320485 \\
\hline C26 & 3. 008165 & 2. 338437 & -0.120993 \\
\hline $\mathrm{C} 27$ & 0.963998 & -2.936609 & -1.166228 \\
\hline C28 & 3. 432560 & -3.664908 & -0.155155 \\
\hline C29 & 1. 355484 & -4.276995 & -1.228809 \\
\hline C30 & 2. 599157 & -4.647084 & -0.707477 \\
\hline C31 & 0.969262 & 2. 947132 & -1.151046 \\
\hline C32 & 3. 438741 & 3. 665935 & -0.135453 \\
\hline C33 & 1. 363025 & 4. 287174 & -1.20646 \\
\hline C34 & 2. 607143 & 4. 652418 & -0.682798 \\
\hline C35 & -1.219828 & -3.173890 & 1. 393857 \\
\hline C36 & -3.607908 & -3.667153 & 0.11247 \\
\hline C37 & -2.966502 & -4.712083 & 0.787760 \\
\hline C38 & -1.758354 & -4.462058 & 1. 44850 \\
\hline
\end{tabular}




\begin{tabular}{|c|c|c|c|}
\hline C39 & -3.625852 & 3. 656324 & 0.088563 \\
\hline $\mathrm{C} 40$ & -1.241380 & 3. 181070 & 1. 383385 \\
\hline C41 & -1.785484 & 4. 467312 & 1. 427570 \\
\hline C42 & -2.991612 & 4. 708227 & 0.759794 \\
\hline H43 & -5.294099 & -2.160973 & -1.522222 \\
\hline H44 & -5.304799 & 2. 131381 & -1.535300 \\
\hline H45 & 5. 554123 & -2.159016 & 1. 180822 \\
\hline H46 & 5.557670 & 2. 149307 & 1. 192635 \\
\hline H47 & 2. 727597 & 0.009213 & -3.044675 \\
\hline H48 & 1. 122981 & 0.010142 & -3.271430 \\
\hline H49 & -0.719777 & -0.002402 & 3. 334408 \\
\hline H50 & -2.327930 & 0.016261 & 3. 164639 \\
\hline H51 & -6.308413 & -0.019483 & -2.257555 \\
\hline H52 & 6. 656709 & -0.007277 & 1. 742449 \\
\hline H53 & 0.699190 & -5.008681 & -1.683253 \\
\hline H54 & 2. 927227 & -5.679544 & -0.743577 \\
\hline H55 & 4. 409951 & -3.932811 & 0.226576 \\
\hline H56 & 0.012232 & -2.592701 & -1.547165 \\
\hline H57 & 0.708098 & 5. 022345 & -1.657237 \\
\hline H58 & 2. 936950 & 5. 684503 & -0.713318 \\
\hline H59 & 4. 416469 & 3. 930206 & 0.247944 \\
\hline H60 & 0.017054 & 2. 606772 & -1.534055 \\
\hline H61 & -3.414652 & -5.698751 & 0.812234 \\
\hline H62 & -1.251653 & -5.242851 & 2. 002603 \\
\hline H63 & -1.284542 & 5. 253607 & 1. 979129 \\
\hline H64 & -3.443799 & 5. 693223 & 0.775852 \\
\hline H65 & -4.558646 & -3.846190 & -0.372983 \\
\hline H66 & -0.287784 & -2.927740 & 1. 888165 \\
\hline H67 & -4.575117 & 3. 828387 & -0.402242 \\
\hline H68 & -0.310756 & 2. 941879 & 1. 883730 \\
\hline
\end{tabular}

\section{Cartesian coordinates of complex $\mathbf{2}$ :}

Number of atoms in complex 2: 78

$\begin{array}{lrrr}\text { Mn1 } & -1.017986 & -0.099747 & 0.745363 \\ \text { Mn2 } & 1.284004 & 0.054226 & -0.682740 \\ 03 & -0.424049 & -0.190284 & -0.998755 \\ 04 & 0.800723 & 0.126945 & 0.990767 \\ 05 & -1.228309 & -0.366240 & 2.804953 \\ \text { 06 } & 1.630476 & 0.150368 & -2.757543 \\ \text { N7 } & -1.943515 & -2.295444 & 0.910659 \\ \text { N8 } & -3.038767 & -0.076196 & 0.044204 \\ \text { N9 } & -1.741458 & 2.042436 & 0.989794 \\ \text { N10 } & 1.842885 & -1.899745 & -0.695831\end{array}$




\begin{tabular}{|c|c|c|c|}
\hline N11 & 3. 222046 & 0.175544 & -0.094778 \\
\hline N12 & 1. 749418 & 2. 109273 & -0.877034 \\
\hline $\mathrm{C} 13$ & -2.970121 & -2.473901 & 0.079367 \\
\hline $\mathrm{C} 14$ & -3.598817 & -1.217125 & -0.413366 \\
\hline C15 & -4.766819 & -1.183925 & -1.178849 \\
\hline C16 & -5.368955 & 0.048070 & -1.436685 \\
\hline $\mathrm{C} 17$ & -4.815392 & 1. 208682 & -0.904389 \\
\hline $\mathrm{C} 18$ & -3.635223 & 1. 114897 & -0.155948 \\
\hline C19 & -2.979722 & 2. 276059 & 0.498799 \\
\hline $\mathrm{C} 20$ & 3. 116425 & -2.160164 & -0.277180 \\
\hline C21 & 3. 867164 & -0.970289 & 0.170612 \\
\hline $\mathrm{C} 22$ & 5. 107728 & -0.944192 & 0.815607 \\
\hline $\mathrm{C} 23$ & 5. 630041 & 0.293984 & 1. 195000 \\
\hline C24 & 4. 927097 & 1. 473118 & 0.926832 \\
\hline C25 & 3. 701404 & 1. 379235 & 0.263994 \\
\hline C26 & 2. 848644 & 2. 495364 & -0.201562 \\
\hline $\mathrm{C} 27$ & 1. 069244 & -2.896644 & -1.155501 \\
\hline C28 & 3. 627666 & -3.455298 & -0.317933 \\
\hline C29 & 1. 530915 & -4.209364 & -1.230999 \\
\hline C30 & 2. 828386 & -4.491917 & -0.805130 \\
\hline C31 & 1. 060266 & 3. 025740 & -1.621469 \\
\hline C32 & 3. 196648 & 3. 854516 & -0.061173 \\
\hline C33 & 1. 352644 & 4. 419642 & -1.514969 \\
\hline C34 & 2. 425339 & 4. 815242 & -0.682452 \\
\hline C35 & -1.476191 & -3.346533 & 1. 634758 \\
\hline C36 & -3.495668 & -3.761039 & -0.199152 \\
\hline C37 & -2.974402 & -4.854601 & 0.457566 \\
\hline C38 & -1.961374 & -4.672000 & 1. 431189 \\
\hline C39 & -3.604227 & 3. 516021 & 0.662574 \\
\hline C40 & -1.099222 & 3. 020353 & 1. 643957 \\
\hline C41 & -1.658730 & 4. 279628 & 1. 855159 \\
\hline C42 & -2.936334 & 4. 527558 & 1. 353757 \\
\hline H43 & -5.219983 & -2.093823 & -1.552453 \\
\hline H44 & -5.293358 & 2. 165821 & -1.074865 \\
\hline H45 & 5. 651739 & -1.856808 & 1. 031062 \\
\hline H46 & 5. 338531 & 2. 430785 & 1. 223929 \\
\hline H47 & 1. 386639 & -0.630513 & -3.284342 \\
\hline H48 & 0.976511 & 0.867385 & -2.983768 \\
\hline H49 & -0.880347 & -1.298608 & 2. 951479 \\
\hline H50 & -0.730657 & 0.226500 & 3. 393759 \\
\hline H51 & -6.277328 & 0.097380 & -2.028922 \\
\hline H52 & 6. 588315 & 0.342267 & 1. 702719 \\
\hline H53 & 0.884286 & -4.985958 & -1.624719 \\
\hline $\mathrm{H} 54$ & 3. 221640 & -5.502440 & -0.859067 \\
\hline
\end{tabular}




$\begin{array}{lrrr}\text { H55 } & 4.644640 & -3.652809 & 0.002356 \\ \text { H56 } & 0.069123 & -2.612175 & -1.462230 \\ \text { H57 } & 2.671504 & 5.868888 & -0.582475 \\ \text { H58 } & 4.073124 & 4.139322 & 0.508839 \\ \text { H59 } & -3.365537 & -5.850129 & 0.265924 \\ \text { H60 } & -1.113496 & 5.035604 & 2.410270 \\ \text { H61 } & -3.415789 & 5.489281 & 1.508493 \\ \text { H62 } & -4.311149 & -3.891231 & -0.899890 \\ \text { H63 } & -4.607316 & 3.689720 & 0.290693 \\ \text { H64 } & -0.106336 & 2.774526 & 2.011182 \\ \text { N65 } & -0.571909 & -3.039765 & 2.606261 \\ \text { C66 } & -1.430603 & -5.704562 & 2.248091 \\ \text { H67 } & -1.778217 & -6.726261 & 2.121434 \\ \text { C68 } & -0.501990 & -5.381548 & 3.211679 \\ \text { H69 } & -0.085254 & -6.135253 & 3.871060 \\ \text { C70 } & -0.117501 & -4.026952 & 3.365353 \\ \text { H71 } & 0.577209 & -3.748357 & 4.154050 \\ \text { C72 } & -0.505335 & 3.396569 & -3.259125 \\ \text { H73 } & -1.189859 & 2.969738 & -3.988214 \\ \text { N74 } & 0.164000 & 2.531072 & -2.509759 \\ \text { C75 } & -0.353042 & 4.802532 & -3.173895 \\ \text { H76 } & 0.544104 & 5.447825 & -3.814821 \\ \text { C77 } & & 5.314216 & -2.310640 \\ \text { H78 } & 6.382526 & -2.249790\end{array}$

Cartesian coordinates of $\mathrm{Mn}^{\mathrm{IV}}$-oxyl radical (antiparallel spin state) in complex 2: Number of atoms in $\mathrm{Mn}^{\mathrm{IV}}$-oxyl radical (antiparallel spin state): 76

$\begin{array}{lrrr}\text { Mn1 } & -1.250991 & -0.149377 & 0.750538 \\ \text { Mn2 } & 1.071171 & 0.049433 & -0.694093 \\ \text { 03 } & -0.611618 & -0.148694 & -1.061573 \\ \text { 04 } & 0.523839 & 0.063536 & 0.980051 \\ \text { 05 } & -1.665477 & -0.140323 & 2.415300 \\ \text { 06 } & 1.538454 & 0.247033 & -2.738360 \\ \text { N7 } & -1.758559 & -2.182052 & 0.755493 \\ \text { N8 } & -3.115473 & -0.155407 & -0.045065 \\ \text { N9 } & -1.754673 & 1.838691 & 0.784719 \\ \text { N10 } & 1.592397 & -1.909164 & -0.810424 \\ \text { N11 } & 2.981911 & 0.078325 & 0.022261 \\ \text { N12 } & 1.631946 & 2.106521 & -0.753514 \\ \text { C13 } & -2.921716 & -2.488925 & 0.150685 \\ \text { C14 } & -3.656689 & -1.333459 & -0.400005 \\ \text { C15 } & -4.844765 & -1.361798 & -1.135121 \\ \text { C16 } & -5.448056 & -0.148015 & -1.474461\end{array}$




\begin{tabular}{|c|c|c|c|}
\hline $\mathrm{C} 17$ & -4.878103 & 1. 061228 & -1.070513 \\
\hline C18 & -3.685290 & 1. 024464 & -0.343669 \\
\hline C19 & -2.950022 & 2. 169306 & 0.224637 \\
\hline $\mathrm{C} 20$ & 2. 822954 & -2.234673 & -0.317163 \\
\hline C21 & 3. 565325 & -1.102688 & 0.272298 \\
\hline C22 & 4. 746310 & -1.159149 & 1. 019178 \\
\hline $\mathrm{C} 23$ & 5. 272166 & 0.035589 & 1. 515363 \\
\hline $\mathrm{C} 24$ & 4. 634076 & 1. 253366 & 1. 256960 \\
\hline C25 & 3. 468586 & 1. 240905 & 0.487452 \\
\hline $\mathrm{C} 26$ & 2. 700302 & 2. 411752 & 0.008603 \\
\hline $\mathrm{C} 27$ & 0.843676 & -2.842196 & -1.420810 \\
\hline C28 & 3. 312234 & -3.534278 & -0.429068 \\
\hline C29 & 1. 287131 & -4.153932 & -1.575937 \\
\hline C30 & 2. 538645 & -4.504792 & -1.069470 \\
\hline C31 & 1. 045467 & 3. 084504 & -1.509148 \\
\hline C32 & 3. 108217 & 3. 745325 & 0.220497 \\
\hline C33 & 1. 409486 & 4. 455502 & -1.341415 \\
\hline C34 & 2. 440034 & 4. 765829 & -0.423407 \\
\hline C35 & -1.113991 & -3.120669 & 1. 517343 \\
\hline C36 & -3.450578 & -3.795580 & 0.166724 \\
\hline C37 & -2.806384 & -4.773502 & 0.894638 \\
\hline C38 & -1.635254 & -4.450691 & 1. 615520 \\
\hline C39 & -3.433735 & 3. 473634 & 0.303007 \\
\hline $\mathrm{C} 40$ & -1.048007 & 2. 748823 & 1. 469372 \\
\hline C41 & -1.489660 & 4. 064648 & 1. 598783 \\
\hline C42 & -2.694171 & 4. 432550 & 0.999160 \\
\hline H43 & -5.299602 & -2.298983 & -1.434125 \\
\hline H44 & -5.356421 & 2. 002236 & -1.317489 \\
\hline H45 & 5. 241572 & -2.101664 & 1. 223546 \\
\hline H46 & 5. 048599 & 2. 176732 & 1. 644513 \\
\hline H47 & 1. 301111 & -0.495583 & -3.320531 \\
\hline H48 & 0.925998 & 0.999988 & -2.963870 \\
\hline H49 & -6.372156 & -0.145814 & -2.044023 \\
\hline H50 & 6. 183019 & 0.019998 & 2. 105700 \\
\hline H51 & 0.663991 & -4.876414 & -2.091962 \\
\hline H52 & 2. 917680 & -5.516012 & -1.181807 \\
\hline H53 & 4. 297387 & -3.782159 & -0.049640 \\
\hline H54 & -0.121550 & -2.506033 & -1.782407 \\
\hline H55 & 2. 737531 & 5. 800233 & -0.273904 \\
\hline H56 & 3. 955674 & 3. 962779 & 0.859837 \\
\hline H57 & -3.216482 & -5.778224 & 0.951230 \\
\hline H58 & -0.908988 & 4. 772810 & 2. 179943 \\
\hline H59 & -3.072217 & 5. 445875 & 1. 094320 \\
\hline H60 & -4.381355 & -4.013820 & -0.343202 \\
\hline
\end{tabular}




$\begin{array}{lrrr}\text { H61 } & -4.392716 & 3.731195 & -0.132734 \\ \text { H62 } & -0.132982 & 2.391103 & 1.926915 \\ \text { N63 } & -0.014083 & -2.712226 & 2.181936 \\ \text { C64 } & -0.961061 & -5.357980 & 2.475787 \\ \text { H65 } & -1.333697 & -6.372839 & 2.583149 \\ \text { C66 } & 0.136128 & -4.918593 & 3.177345 \\ \text { H67 } & 0.666085 & -5.568246 & 3.865806 \\ \text { C68 } & 0.570048 & -3.580322 & 2.991075 \\ \text { H69 } & 1.434889 & -3.214027 & 3.540054 \\ \text { C70 } & -0.387635 & 3.595169 & -3.230700 \\ \text { H71 } & -1.049766 & 3.230041 & -4.012178 \\ \text { N72 } & 0.176766 & 2.670890 & -2.464287 \\ \text { C73 } & -0.153399 & 4.985621 & -3.098305 \\ \text { H74 } & -0.658402 & 5.682245 & -3.758856 \\ \text { C75 } & 0.756530 & 5.415408 & -2.159879 \\ \text { H76 } & 1.004874 & 6.468063 & -2.055619\end{array}$

Cartesian coordinates of $\mathrm{Mn}^{\mathrm{IV}}$-oxyl radical (parallel spin state) in complex 2:

Number of atoms in $\mathrm{Mn}^{\mathrm{IV}}$-oxyl radical (parallel spin state): 76

$\begin{array}{lrrr}\text { Mn1 } & -1.256190 & -0.151786 & 0.615194 \\ \text { Mn2 } & 1.106207 & 0.049345 & -0.718476 \\ \text { 03 } & -0.580245 & -0.152131 & -1.129272 \\ \text { 04 } & 0.515232 & 0.063712 & 0.932248 \\ \text { 05 } & -1.847851 & -0.123411 & 2.334978 \\ \text { 06 } & 1.602714 & 0.250804 & -2.761265 \\ \text { N7 } & -1.779457 & -2.167220 & 0.735938 \\ \text { N8 } & -3.114384 & -0.157670 & -0.139844 \\ \text { N9 } & -1.760618 & 1.799041 & 0.766575 \\ \text { N10 } & 1.630625 & -1.907285 & -0.822764 \\ \text { N11 } & 3.001544 & 0.085953 & 0.024199 \\ \text { N12 } & 1.656451 & 2.109841 & -0.771598 \\ \text { C13 } & -2.950969 & -2.479984 & 0.140178 \\ \text { C14 } & -3.686665 & -1.334828 & -0.433246 \\ \text { C15 } & -4.913368 & -1.359749 & -1.104457 \\ \text { C16 } & -5.520234 & -0.142545 & -1.425975 \\ \text { C17 } & -4.930774 & 1.066340 & -1.046798 \\ \text { C18 } & -3.701536 & 1.024747 & -0.383094 \\ \text { C19 } & -2.957872 & 2.150387 & 0.212798 \\ \text { C20 } & 2.853773 & -2.229283 & -0.309741 \\ \text { C21 } & 3.586123 & -1.093097 & 0.283340 \\ \text { C22 } & 4.760184 & -1.143586 & 1.041242 \\ \text { C23 } & & & 1.538223 \\ \text { C24 } & 0.054027 & 1.269729\end{array}$




\begin{tabular}{|c|c|c|c|}
\hline C25 & 3. 481247 & 1. 251745 & 0.489867 \\
\hline C26 & 2. 715068 & 2. 419864 & 0.001582 \\
\hline $\mathrm{C} 27$ & 0.890228 & -2.843971 & -1.437754 \\
\hline C28 & 3. 344893 & -3.529284 & -0.407032 \\
\hline C29 & 1. 335932 & -4.156674 & -1.577992 \\
\hline C30 & 2. 580221 & -4.504012 & -1.051856 \\
\hline C31 & 1. 070806 & 3. 085054 & -1.531076 \\
\hline C32 & 3. 114830 & 3. 755166 & 0.217510 \\
\hline C33 & 1. 425353 & 4. 457954 & -1.358158 \\
\hline C34 & 2. 446774 & 4. 772676 & -0.431553 \\
\hline C35 & -1.128502 & -3.105405 & 1. 498329 \\
\hline C36 & -3.489678 & -3.780572 & 0.187135 \\
\hline C37 & -2.853132 & -4.747829 & 0.935965 \\
\hline C38 & -1.669619 & -4.424452 & 1. 634771 \\
\hline C39 & -3.437034 & 3. 452076 & 0.335379 \\
\hline $\mathrm{C} 40$ & -1.055998 & 2. 685178 & 1. 488142 \\
\hline C41 & -1.500795 & 3. 993345 & 1. 666655 \\
\hline C42 & -2.701183 & 4. 383522 & 1. 072664 \\
\hline H43 & -5.398279 & -2.294189 & -1.361873 \\
\hline H44 & -5.426846 & 2. 007247 & -1.256602 \\
\hline H45 & 5. 256158 & -2.084132 & 1. 252772 \\
\hline H46 & 5. 048478 & 2. 195022 & 1. 657309 \\
\hline H47 & 1. 392752 & -0.490610 & -3.355333 \\
\hline $\mathrm{H} 48$ & 0.987204 & 0.996802 & -2.997556 \\
\hline H49 & -6.471658 & -0.137299 & -1.948509 \\
\hline H50 & 6. 184108 & 0.042787 & 2. 136694 \\
\hline H51 & 0.719943 & -4.882508 & -2.097897 \\
\hline H52 & 2. 960746 & -5.515921 & -1.152274 \\
\hline H53 & 4. 324704 & -3.774804 & -0.012537 \\
\hline H54 & -0.069432 & -2.510840 & -1.816723 \\
\hline H55 & 2. 737496 & 5. 808492 & -0.278569 \\
\hline H56 & 3. 955339 & 3. 976753 & 0.864561 \\
\hline H57 & -3.275649 & -5.745426 & 1. 020662 \\
\hline H58 & -0.923809 & 4. 679520 & 2. 277053 \\
\hline H59 & -3.078955 & 5. 393176 & 1. 202030 \\
\hline H60 & -4.422818 & -4.002406 & -0.316744 \\
\hline H61 & -4.391209 & 3. 728367 & -0.099433 \\
\hline H62 & -0.139234 & 2. 311750 & 1. 928783 \\
\hline N63 & 0.001122 & -2.709982 & 2. 118144 \\
\hline C64 & -0.995316 & -5.326154 & 2. 500828 \\
\hline H65 & -1.385708 & -6.330640 & 2. 638163 \\
\hline C66 & 0.127332 & -4.894839 & 3. 165365 \\
\hline H67 & 0.659936 & -5.539181 & 3. 856756 \\
\hline C68 & 0.588740 & -3.573858 & 2. 928952 \\
\hline
\end{tabular}




$\begin{array}{lrrr}\text { H69 } & 1.482179 & -3.218458 & 3.438001 \\ \text { C70 } & -0.352428 & 3.589338 & -3.262302 \\ \text { H71 } & -1.006952 & 3.221542 & -4.048923 \\ \text { N72 } & 0.212320 & 2.667226 & -2.493561 \\ \text { C73 } & -0.127745 & 4.981121 & -3.125755 \\ \text { H74 } & -0.632512 & 5.675686 & -3.788656 \\ \text { C75 } & 0.772652 & 5.415148 & -2.180132 \\ \text { H76 } & 1.013553 & 6.469173 & -2.072376\end{array}$

Cartesian coordinates of reactant state associated with one water molecule approaching the antiparallel $\mathrm{Mn}^{\mathrm{IV}}$-oxyl radical in complex 2:

Number of atoms in reactant-state complex: 79

\begin{tabular}{|c|c|c|c|}
\hline Mn1 & -1.243593 & -0.128459 & 0.763663 \\
\hline Mn2 & 1. 107223 & 0.050393 & -0.640654 \\
\hline 03 & -0.579353 & -0.149379 & -1.021768 \\
\hline 04 & 0.527163 & 0.086435 & 1. 027881 \\
\hline 05 & -1.691895 & -0.101693 & 2. 430100 \\
\hline 06 & 1. 579129 & 0.231856 & -2.680126 \\
\hline N7 & -1.752207 & -2.165340 & 0.804150 \\
\hline N8 & -3.113536 & -0.144650 & -0.017835 \\
\hline N9 & -1.744620 & 1. 857995 & 0.782124 \\
\hline N10 & 1. 623657 & -1.907566 & -0.744761 \\
\hline N11 & 3. 011345 & 0.079744 & 0.072318 \\
\hline N12 & 1. 661816 & 2. 101639 & -0.711139 \\
\hline $\mathrm{C} 13$ & -2.924672 & -2.475653 & 0.216769 \\
\hline C14 & -3.668835 & -1.326198 & -0.334351 \\
\hline C15 & -4.876587 & -1.362851 & -1.036996 \\
\hline C16 & -5.484407 & -0.152966 & -1.381633 \\
\hline $\mathrm{C} 17$ & -4.902002 & 1. 060952 & -1.010730 \\
\hline C18 & -3.690863 & 1. 031694 & -0.314429 \\
\hline C19 & -2.945586 & 2. 182078 & 0.228803 \\
\hline $\mathrm{C} 20$ & 2. 857169 & -2.231182 & -0.256508 \\
\hline C21 & 3. 606888 & -1.096161 & 0.312441 \\
\hline $\mathrm{C} 22$ & 4. 807616 & -1.145756 & 1. 025622 \\
\hline C23 & 5. 339938 & 0.050078 & 1. 505911 \\
\hline $\mathrm{C} 24$ & 4. 688336 & 1. 263700 & 1. 263755 \\
\hline $\mathrm{C} 25$ & 3. 504498 & 1. 244936 & 0.525112 \\
\hline C26 & 2. 730283 & 2. 411157 & 0.050053 \\
\hline $\mathrm{C} 27$ & 0.870252 & -2.842630 & -1.347890 \\
\hline C28 & 3. 340794 & -3.533886 & -0.360173 \\
\hline C29 & 1. 310013 & -4.155547 & -1.496499 \\
\hline C30 & 2. 562254 & -4.506301 & -0.990262 \\
\hline C31 & 1. 076130 & 3. 074105 & -1.475494 \\
\hline
\end{tabular}




\begin{tabular}{|c|c|c|c|}
\hline C32 & 3. 132030 & 3. 747988 & 0.257687 \\
\hline C33 & 1. 436725 & 4. 446290 & -1.313773 \\
\hline C34 & 2. 462993 & 4. 763342 & -0.392309 \\
\hline C35 & -1.082406 & -3.109545 & 1. 537497 \\
\hline C36 & -3.448071 & -3.783786 & 0.238730 \\
\hline C37 & -2.782380 & -4.764600 & 0.943161 \\
\hline C38 & -1.592163 & -4.444478 & 1. 632043 \\
\hline C39 & -3.424693 & 3. 488985 & 0.291237 \\
\hline $\mathrm{C} 40$ & -1.028159 & 2. 778898 & 1. 442518 \\
\hline C41 & -1.466038 & 4. 097233 & 1. 556001 \\
\hline C42 & -2.676468 & 4. 457636 & 0.963963 \\
\hline H43 & -5.343665 & -2.302853 & -1.306609 \\
\hline H44 & -5.386094 & 1. 998962 & -1.257791 \\
\hline H45 & 5. 308407 & -2.086558 & 1. 222204 \\
\hline H46 & 5. 108906 & 2. 190304 & 1. 636846 \\
\hline H47 & 1. 342274 & -0.517472 & -3.254050 \\
\hline H48 & 0.966747 & 0.982990 & -2.914270 \\
\hline H49 & -6.422963 & -0.157226 & -1.927011 \\
\hline H50 & 6. 265850 & 0. 039969 & 2. 072172 \\
\hline H51 & 0.683931 & -4.879567 & -2.006737 \\
\hline H52 & 2. 937683 & -5.519587 & -1.095970 \\
\hline H53 & 4. 325745 & -3.781725 & 0. 019369 \\
\hline H54 & -0.094968 & -2.506288 & -1.709270 \\
\hline H55 & 2. 757135 & 5. 799251 & -0.246514 \\
\hline H56 & 3. 976834 & 3. 970060 & 0.898916 \\
\hline H57 & -3.185655 & -5.772059 & 0.999842 \\
\hline H58 & -0.876690 & 4. 813842 & 2. 117709 \\
\hline H59 & -3.051327 & 5. 473308 & 1. 045770 \\
\hline H60 & -4.387820 & -4.003712 & -0.253494 \\
\hline H61 & -4.386682 & 3. 742182 & -0.140319 \\
\hline H62 & -0.105943 & 2. 428630 & 1. 890997 \\
\hline N63 & 0.039851 & -2.710248 & 2. 169953 \\
\hline C64 & -0.880558 & -5.364078 & 2. 448537 \\
\hline H65 & -1.246361 & -6.381847 & 2. 552298 \\
\hline C66 & 0.247617 & -4.936065 & 3. 106157 \\
\hline H67 & 0.811429 & -5.598752 & 3. 754147 \\
\hline C68 & 0.670758 & -3.593555 & 2. 929094 \\
\hline H69 & 1. 564027 & -3.219404 & 3. 424165 \\
\hline $\mathrm{C} 70$ & -0.348785 & 3. 572022 & -3.207861 \\
\hline H71 & -1.005713 & 3. 200623 & -3.990753 \\
\hline N72 & 0.213665 & 2. 653282 & -2.432691 \\
\hline C73 & -0.117892 & 4. 963109 & -3.082268 \\
\hline H74 & -0.620122 & 5. 655136 & -3.749707 \\
\hline C75 & 0.786523 & 5. 399808 & -2.141165 \\
\hline
\end{tabular}




$\begin{array}{rrrr}\text { H76 } & 1.032770 & 6.453474 & -2.042074 \\ \text { 077 } & 2.350295 & -0.919899 & 3.128565 \\ \text { H78 } & 1.460806 & -0.863722 & 2.737612 \\ \text { H79 } & 2.245728 & -0.621047 & 4.045612\end{array}$

Cartesian coordinates of transition state associated with one water molecule approaching the antiparallel $\mathrm{Mn}^{\mathrm{IV}}$-oxyl radical (TS1) in complex 2:

Number of atoms in transition-state complex: 79

\begin{tabular}{|c|c|c|c|}
\hline Mn1 & -1.248776 & -0.076592 & 0.839380 \\
\hline Mn2 & 1. 062097 & 0.057200 & -0.628115 \\
\hline 03 & -0.613665 & -0.125179 & -0.995300 \\
\hline 04 & 0.567778 & 0.093941 & 1. 073729 \\
\hline 05 & -1.631619 & 0.010277 & 2. 563572 \\
\hline 06 & 1. 479701 & 0.226589 & -2.689920 \\
\hline N7 & -1.807128 & -2.211978 & 0.925732 \\
\hline N8 & -3.110892 & -0.135154 & 0.081125 \\
\hline N9 & -1.723505 & 1. 892524 & 0.787766 \\
\hline N10 & 1. 617214 & -1.897188 & -0.724745 \\
\hline N11 & 3. 022525 & 0.132636 & 0.007516 \\
\hline N12 & 1. 655324 & 2. 157417 & -0.776337 \\
\hline $\mathrm{C} 13$ & -2.902773 & -2.487801 & 0.194841 \\
\hline C14 & -3.630788 & -1.310139 & -0.320560 \\
\hline $\mathrm{C} 15$ & -4.800917 & -1.341805 & -1.083639 \\
\hline $\mathrm{C} 16$ & -5.421015 & -0.134935 & -1.415702 \\
\hline $\mathrm{C} 17$ & -4.865301 & 1. 070668 & -0.993023 \\
\hline $\mathrm{C} 18$ & -3.683834 & 1. 037629 & -0.244648 \\
\hline C19 & -2.946450 & 2. 197921 & 0.277604 \\
\hline $\mathrm{C} 20$ & 2. 880035 & -2.189591 & -0.297270 \\
\hline $\mathrm{C} 21$ & 3. 634030 & -1.037320 & 0.240786 \\
\hline C22 & 4. 855530 & -1.074018 & 0.920830 \\
\hline $\mathrm{C} 23$ & 5. 399026 & 0.133167 & 1. 365639 \\
\hline $\mathrm{C} 24$ & 4. 738595 & 1. 340509 & 1. 118668 \\
\hline $\mathrm{C} 25$ & 3. 530156 & 1. 306476 & 0.416639 \\
\hline $\mathrm{C} 26$ & 2. 743668 & 2. 472484 & -0.052489 \\
\hline $\mathrm{C} 27$ & 0.860529 & -2.852841 & -1.290248 \\
\hline C28 & 3. 393386 & -3.479083 & -0.427526 \\
\hline C29 & 1. 326061 & -4.154672 & -1.460994 \\
\hline C30 & 2. 611171 & -4.472107 & -1.020339 \\
\hline C31 & 1. 053548 & 3. 123520 & -1.531317 \\
\hline C32 & 3. 161045 & 3. 809020 & 0.127470 \\
\hline C33 & 1. 421806 & 4. 497099 & -1.399015 \\
\hline C34 & 2. 476329 & 4. 820658 & -0.513310 \\
\hline C35 & -1.240032 & -3.220824 & 1. 655878 \\
\hline
\end{tabular}




\begin{tabular}{|c|c|c|c|}
\hline C36 & -3.382534 & -3.805631 & 0.009716 \\
\hline C37 & -2.749934 & -4.848651 & 0.642545 \\
\hline C38 & -1.670694 & -4.575891 & 1. 516428 \\
\hline C39 & -3.446037 & 3. 499023 & 0.338211 \\
\hline $\mathrm{C} 40$ & -0.996464 & 2. 843698 & 1. 392120 \\
\hline C41 & -1.444336 & 4. 157933 & 1. 496994 \\
\hline C42 & -2.687008 & 4. 491607 & 0.957103 \\
\hline H43 & -5.235195 & -2.278498 & -1.411352 \\
\hline H44 & -5.337566 & 2. 012208 & -1.248999 \\
\hline H45 & 5. 370646 & -2.008835 & 1. 110968 \\
\hline H46 & 5. 170827 & 2.274325 & 1. 458861 \\
\hline H47 & 1. 165444 & -0.513162 & -3.238256 \\
\hline H48 & 0.881026 & 0.998801 & -2.893001 \\
\hline H49 & -6.334923 & -0.138074 & -2.001080 \\
\hline H50 & 6. 342634 & 0.135191 & 1. 902325 \\
\hline H51 & 0.695302 & -4.894585 & -1.941829 \\
\hline H52 & 3. 009252 & -5.474055 & -1.148873 \\
\hline H53 & 4. 403736 & -3.700433 & -0.101946 \\
\hline H54 & -0.127647 & -2.539551 & -1.607613 \\
\hline H55 & 2. 778599 & 5.856843 & -0.387753 \\
\hline H56 & 4. 025651 & 4. 036619 & 0.739739 \\
\hline H57 & -3.099621 & -5.869650 & 0.517334 \\
\hline H58 & -0.837815 & 4. 894220 & 2. 013114 \\
\hline H59 & -3.072962 & 5.503062 & 1. 037512 \\
\hline H60 & -4.254530 & -3.989637 & -0.605515 \\
\hline H61 & -4.430457 & 3. 727087 & -0.054864 \\
\hline H62 & -0.047832 & 2. 524369 & 1. 805581 \\
\hline N63 & -0.283984 & -2.883285 & 2.563092 \\
\hline C64 & -1.034715 & -5.572605 & 2. 300974 \\
\hline H65 & -1.351508 & -6.607815 & 2. 207600 \\
\hline C66 & -0.051451 & -5.206216 & 3. 191400 \\
\hline H67 & 0.444305 & -5.932862 & 3. 825654 \\
\hline C68 & 0.270843 & -3.837495 & 3. 303711 \\
\hline H69 & 0.994840 & -3.506715 & 4. 044400 \\
\hline C70 & -0.418506 & 3. 600625 & -3.229255 \\
\hline H71 & -1.097404 & 3. 221620 & -3.989477 \\
\hline N72 & 0.163197 & 2. 691371 & -2.459299 \\
\hline $\mathrm{C} 73$ & -0.182550 & 4. 994005 & -3.126893 \\
\hline H74 & -0.703953 & 5. 678043 & -3.787839 \\
\hline C75 & 0.748756 & 5. 441981 & -2.218585 \\
\hline H76 & 0.998486 & 6. 496540 & -2.140241 \\
\hline 077 & -0.296915 & -0.443653 & 3. 469551 \\
\hline H78 & -0.254677 & -1.442160 & 3. 140198 \\
\hline H79 & 0.391474 & -0.002233 & 2. 911512 \\
\hline
\end{tabular}


Cartesian coordinates of product state associated with one water molecule approaching the antiparallel $\mathrm{Mn}^{\mathrm{IV}}$-oxyl radical in complex 2:

Number of atoms in product-state complex: 79

\begin{tabular}{|c|c|c|c|}
\hline Mn1 & -1.098445 & -0.017814 & 0.820678 \\
\hline Mn2 & 1. 243450 & 0.115793 & -0.623116 \\
\hline 03 & -0.445762 & -0.197702 & -0.925455 \\
\hline 04 & 0.818194 & 0.252929 & 1. 048685 \\
\hline 05 & -1.324075 & -0.096584 & 2. 667849 \\
\hline 06 & 1. 564032 & 0. 189485 & -2.725094 \\
\hline N7 & -1.951855 & -2.404061 & 0.962208 \\
\hline N8 & -3.003091 & -0.117473 & 0.086673 \\
\hline N9 & -1.595536 & 1. 916207 & 0.934096 \\
\hline N10 & 1. 884974 & -1.814010 & -0.632776 \\
\hline N11 & 3. 218361 & 0.313517 & -0.090545 \\
\hline N12 & 1. 704140 & 2. 216871 & -0.898277 \\
\hline $\mathrm{C} 13$ & -3.011686 & -2.519285 & 0. 153219 \\
\hline $\mathrm{C} 14$ & -3.584277 & -1.248497 & -0.364131 \\
\hline C15 & -4.729266 & -1.189320 & -1.165270 \\
\hline $\mathrm{C} 16$ & -5.282427 & 0.055990 & -1.468464 \\
\hline $\mathrm{C} 17$ & -4.696200 & 1. 210983 & -0.960913 \\
\hline $\mathrm{C} 18$ & -3.539291 & 1. 089766 & -0.182049 \\
\hline C19 & -2.816734 & 2. 226682 & 0.430406 \\
\hline $\mathrm{C} 20$ & 3. 178243 & -2.025444 & -0.252560 \\
\hline C21 & 3. 898536 & -0.809303 & 0. 179452 \\
\hline $\mathrm{C} 22$ & 5. 146737 & -0.747419 & 0.806681 \\
\hline $\mathrm{C} 23$ & 5.644195 & 0.508165 & 1. 162089 \\
\hline C24 & 4. 910917 & 1. 664793 & 0.881730 \\
\hline $\mathrm{C} 25$ & 3. 678149 & 1. 531712 & 0.236298 \\
\hline C26 & 2. 805081 & 2. 627617 & -0.246197 \\
\hline $\mathrm{C} 27$ & 1. 145159 & -2.835351 & -1.094780 \\
\hline C28 & 3. 742411 & -3.298359 & -0.325559 \\
\hline C29 & 1. 658993 & -4.125682 & -1.204480 \\
\hline C30 & 2. 977296 & -4.359761 & -0.812003 \\
\hline C31 & 1. 001463 & 3. 112018 & -1.652494 \\
\hline C32 & 3. 148558 & 3. 993006 & -0.143471 \\
\hline C33 & 1. 289315 & 4. 509801 & -1.593049 \\
\hline C34 & 2. 368928 & 4. 933450 & -0.783147 \\
\hline C35 & -1.593496 & -3.489917 & 1. 676483 \\
\hline C36 & -3.635396 & -3.773284 & -0.101982 \\
\hline C37 & -3.201740 & -4.899686 & 0.554265 \\
\hline C38 & -2.169543 & -4.780754 & 1. 520354 \\
\hline C39 & -3.345925 & 3. 509235 & 0.573879 \\
\hline
\end{tabular}




\begin{tabular}{|c|c|c|c|}
\hline $\mathrm{C} 40$ & -0.891052 & 2. 835393 & 1. 608857 \\
\hline C41 & -1.366024 & 4. 131810 & 1. 796571 \\
\hline C42 & -2.610456 & 4. 472819 & 1. 267062 \\
\hline H43 & -5.202452 & -2.086413 & -1.543790 \\
\hline H44 & -5.124925 & 2. 182701 & -1.176470 \\
\hline H45 & 5. 716161 & -1.643673 & 1. 025523 \\
\hline H46 & 5. 305030 & 2. 636461 & 1. 155701 \\
\hline H47 & 1. 273634 & -0.595567 & -3.220280 \\
\hline H48 & 0.907510 & 0.907300 & -2.934555 \\
\hline H49 & -6.172962 & 0.119444 & -2.085764 \\
\hline H50 & 6. 607134 & 0.587257 & 1. 656842 \\
\hline H51 & 1. 040376 & -4.921768 & -1.604673 \\
\hline H52 & 3. 413030 & -5.350667 & -0.896047 \\
\hline H53 & 4. 775611 & -3.456000 & -0.036644 \\
\hline H54 & 0.130229 & -2.580346 & -1.379203 \\
\hline H55 & 2. 611771 & 5.990380 & -0.715226 \\
\hline H56 & 4. 028179 & 4. 297383 & 0.411269 \\
\hline H57 & -3.669853 & -5.863434 & 0.376072 \\
\hline H58 & -0.780920 & 4. 845884 & 2. 365936 \\
\hline H59 & -3.018531 & 5. 468653 & 1. 410178 \\
\hline H60 & -4.466079 & -3.846476 & -0.792008 \\
\hline H61 & -4.331408 & 3. 748524 & 0.190965 \\
\hline H62 & 0.058689 & 2. 498200 & 2. 005968 \\
\hline N63 & -0.652179 & -3.308112 & 2. 655437 \\
\hline C64 & -1.722911 & -5.834750 & 2. 355719 \\
\hline H65 & -2.157944 & -6.824473 & 2. 244572 \\
\hline C66 & -0.765501 & -5.594155 & 3. 324991 \\
\hline H67 & -0.424236 & -6.376234 & 3. 993597 \\
\hline C68 & -0.260498 & -4.294597 & 3. 465648 \\
\hline H69 & 0.448834 & -4.025215 & 4. 241453 \\
\hline C70 & -0.591279 & 3. 430177 & -3.276318 \\
\hline H71 & -1.287205 & 2. 980406 & -3.980641 \\
\hline N72 & 0.088098 & 2. 589412 & -2.509300 \\
\hline C73 & -0.437869 & 4. 838178 & -3.240643 \\
\hline H74 & -1.037384 & 5. 462157 & -3.894718 \\
\hline C75 & 0.513095 & 5. 377999 & -2.405867 \\
\hline H76 & 0.699774 & 6. 448062 & -2.380425 \\
\hline 077 & -0.175853 & -0.775452 & 3. 253211 \\
\hline H78 & -0.352978 & -2.300435 & 2. 853519 \\
\hline H79 & 0.572620 & -0.328160 & 2. 790903 \\
\hline
\end{tabular}

Cartesian coordinates of reactant state associated with one water molecule approaching the parallel $\mathrm{Mn}^{\mathrm{IV}}$-oxyl radical in complex 2: 
Number of atoms in reactant-state complex: 79

\begin{tabular}{|c|c|c|c|}
\hline Mn1 & -1.252301 & -0.155268 & 0. 605931 \\
\hline Mn2 & 1. 117789 & 0.024428 & -0.729755 \\
\hline 03 & -0.576808 & -0.165764 & -1.132715 \\
\hline 04 & 0.518934 & 0.048335 & 0.924526 \\
\hline 05 & -1.821385 & -0.131403 & 2. 336360 \\
\hline 06 & 1. 595328 & 0.221046 & -2.771413 \\
\hline N7 & -1.780642 & -2.169068 & 0.735553 \\
\hline N8 & -3.119059 & -0.153091 & -0.125223 \\
\hline N9 & -1.742019 & 1. 800304 & 0.754157 \\
\hline N10 & 1. 627448 & -1.934285 & -0.839838 \\
\hline N11 & 3. 015900 & 0.047887 & -0.012983 \\
\hline N12 & 1. 669468 & 2. 076176 & -0.785427 \\
\hline C13 & -2.960530 & -2.476951 & 0.152511 \\
\hline C14 & -3.703172 & -1.328120 & -0.403670 \\
\hline C15 & -4.943409 & -1.348762 & -1.049633 \\
\hline $\mathrm{C} 16$ & -5.551115 & -0.129620 & -1.361952 \\
\hline $\mathrm{C} 17$ & -4.948289 & 1. 077233 & -0.998012 \\
\hline C18 & -3.706196 & 1. 031331 & -0.359010 \\
\hline C19 & -2.946465 & 2. 155347 & 0.218664 \\
\hline C20 & 2. 858335 & -2.262562 & -0.348557 \\
\hline $\mathrm{C} 21$ & 3. 609618 & -1.130201 & 0. 222997 \\
\hline C22 & 4. 810187 & -1.184092 & 0.935882 \\
\hline $\mathrm{C} 23$ & 5. 344671 & 0.008942 & 1. 420526 \\
\hline $\mathrm{C} 24$ & 4. 694760 & 1. 224345 & 1. 183186 \\
\hline C25 & 3. 510841 & 1. 210802 & 0.444788 \\
\hline C26 & 2. 737987 & 2. 380715 & -0.022794 \\
\hline $\mathrm{C} 27$ & 0.870949 & -2.866484 & -1.443652 \\
\hline C28 & 3. 337052 & -3.567153 & -0.450894 \\
\hline C29 & 1. 305501 & -4.181392 & -1.590075 \\
\hline C30 & 2. 555486 & -4.536812 & -1.081568 \\
\hline C31 & 1. 080090 & 3. 053700 & -1.540171 \\
\hline C32 & 3. 139210 & 3. 716124 & 0.194109 \\
\hline C33 & 1. 438695 & 4. 425109 & -1.367321 \\
\hline C34 & 2. 467266 & 4. 735791 & -0.446306 \\
\hline C35 & -1.111320 & -3.119567 & 1. 465679 \\
\hline C36 & -3.494227 & -3.779455 & 0.187234 \\
\hline C37 & -2.836336 & -4.759891 & 0.899814 \\
\hline C38 & -1.638020 & -4.446842 & 1. 576975 \\
\hline C39 & -3.415973 & 3. 460799 & 0.339707 \\
\hline $\mathrm{C} 40$ & -1.018645 & 2. 689301 & 1. 453223 \\
\hline C41 & -1.452144 & 4. 001495 & 1. 629210 \\
\hline C42 & -2.661684 & 4. 393897 & 1. 055613 \\
\hline H43 & -5.438132 & -2.281358 & -1.294572 \\
\hline
\end{tabular}




\begin{tabular}{|c|c|c|c|}
\hline H44 & -5.443980 & 2. 019984 & -1.200184 \\
\hline H45 & 5. 309400 & -2.126516 & 1. 128635 \\
\hline H46 & 5. 116681 & 2. 148960 & 1. 559634 \\
\hline H47 & 1. 373352 & -0.522755 & -3.358309 \\
\hline H48 & 0.980826 & 0.969945 & -3.003927 \\
\hline H49 & -6.512976 & -0.121223 & -1.864979 \\
\hline H50 & 6. 270741 & -0.004772 & 1. 986459 \\
\hline H51 & 0.677138 & -4.903258 & -2.100568 \\
\hline H52 & 2. 926816 & -5.551760 & -1.185757 \\
\hline H53 & 4. 320232 & -3.818901 & -0.069350 \\
\hline H54 & -0.091836 & -2.527006 & -1.808726 \\
\hline H55 & 2. 760411 & 5. 770867 & -0.292821 \\
\hline H56 & 3. 985046 & 3. 934254 & 0.835340 \\
\hline H57 & -3.250243 & -5.762286 & 0.968699 \\
\hline H58 & -0.858620 & 4. 689720 & 2. 221153 \\
\hline H59 & -3.031010 & 5. 406868 & 1. 183371 \\
\hline H60 & -4.435776 & -3.995911 & -0.303052 \\
\hline H61 & -4.375848 & 3. 739332 & -0.080839 \\
\hline H62 & -0.093920 & 2. 315861 & 1. 876526 \\
\hline N63 & 0.029085 & -2.735787 & 2. 073731 \\
\hline C64 & -0.930640 & -5.371075 & 2. 391871 \\
\hline H65 & -1.311201 & -6.382006 & 2. 508374 \\
\hline C66 & 0.213657 & -4.956241 & 3. 029183 \\
\hline H67 & 0.775860 & -5.621935 & 3. 675477 \\
\hline C68 & 0.657660 & -3.623918 & 2. 829173 \\
\hline H69 & 1. 566862 & -3.261844 & 3. 303851 \\
\hline $\mathrm{C} 70$ & -0.351371 & 3. 563582 & -3.263278 \\
\hline H71 & -1.010474 & 3. 197845 & -4.047010 \\
\hline N72 & 0.215744 & 2. 639141 & -2.498349 \\
\hline C73 & -0.122888 & 4. 954074 & -3.126036 \\
\hline H74 & -0.629044 & 5. 650904 & -3.785464 \\
\hline C75 & 0.783850 & 5. 384499 & -2.184286 \\
\hline H76 & 1. 028124 & 6. 437789 & -2.076769 \\
\hline 077 & 2. 335281 & -0.952736 & 3. 023370 \\
\hline H78 & 1. 442887 & -0.915691 & 2. 636019 \\
\hline H79 & 2. 224591 & -0.675614 & 3. 946474 \\
\hline
\end{tabular}

Cartesian coordinates of transition state associated with one water molecule approaching the parallel $\mathrm{Mn}^{\mathrm{IV}}$-oxyl radical (TS2) in complex 2:

Number of atoms in transition-state complex: 79

$\begin{array}{lrrr}\text { Mn1 } & -1.143199 & -0.003252 & 0.804685 \\ \text { Mn2 } & 1.152780 & 0.048416 & -0.676767 \\ 03 & -0.528070 & -0.177483 & -1.011369\end{array}$




\begin{tabular}{|c|c|c|c|}
\hline 04 & 0.646922 & 0.148654 & 1. 006738 \\
\hline 05 & -1.549847 & 0.188388 & 2. 572331 \\
\hline 06 & 1.578196 & 0.146973 & -2.743231 \\
\hline N7 & -1.844950 & -2.197833 & 0.908854 \\
\hline N8 & -3.098385 & -0.046825 & 0.066344 \\
\hline N9 & -1.725092 & 2. 010673 & 0.837783 \\
\hline N10 & 1. 700407 & -1.910817 & -0.698687 \\
\hline N11 & 3. 082446 & 0. 139772 & -0.015538 \\
\hline N12 & 1. 680146 & 2. 106595 & -0.854837 \\
\hline C13 & -2.951400 & -2.422358 & 0. 187479 \\
\hline C14 & -3.661178 & -1.212721 & -0.297367 \\
\hline C15 & -4.867993 & -1.224075 & -1.004693 \\
\hline C16 & -5.478065 & -0.007894 & -1.315289 \\
\hline $\mathrm{C} 17$ & -4.889125 & 1. 188858 & -0.910698 \\
\hline C18 & -3.679547 & 1. 134215 & -0.211873 \\
\hline C19 & -2.945307 & 2. 299762 & 0.324173 \\
\hline C20 & 2. 955209 & -2.189661 & -0.238932 \\
\hline C21 & 3. 696501 & -1.017182 & 0.269397 \\
\hline C22 & 4. 902232 & -1.018063 & 0.977715 \\
\hline C23 & 5. 421427 & 0.208225 & 1. 398635 \\
\hline $\mathrm{C} 24$ & 4. 752225 & 1. 400686 & 1. 104916 \\
\hline C25 & 3. 561970 & 1. 331430 & 0.376927 \\
\hline C26 & 2. 757185 & 2. 465739 & -0.130783 \\
\hline C27 & 0.945872 & -2.887231 & -1.228259 \\
\hline C28 & 3. 465524 & -3.484508 & -0.305734 \\
\hline C29 & 1. 408407 & -4.197289 & -1.335433 \\
\hline C30 & 2. 686521 & -4.499381 & -0.865462 \\
\hline C31 & 1. 053572 & 3. 038952 & -1.634114 \\
\hline C32 & 3. 138297 & 3. 815656 & 0. 018469 \\
\hline C33 & 1. 386923 & 4. 423747 & -1.528454 \\
\hline C34 & 2. 430313 & 4. 793972 & -0.648041 \\
\hline C35 & -1.251865 & -3.243908 & 1. 554400 \\
\hline C36 & -3.461369 & -3.725024 & -0.034361 \\
\hline C37 & -2.826786 & -4.803205 & 0.535891 \\
\hline C38 & -1.705238 & -4.587161 & 1. 371081 \\
\hline C39 & -3.458446 & 3. 595035 & 0.400381 \\
\hline $\mathrm{C} 40$ & -1.012232 & 2. 948281 & 1. 475291 \\
\hline C41 & -1.472032 & 4. 258321 & 1. 592605 \\
\hline C42 & -2.711224 & 4. 585245 & 1. 040675 \\
\hline H43 & -5.339509 & -2.152147 & -1.303070 \\
\hline H44 & -5.363923 & 2. 136390 & -1.137481 \\
\hline H45 & 5. 421549 & -1.940967 & 1. 209924 \\
\hline H46 & 5. 162192 & 2. 348582 & 1. 433578 \\
\hline H47 & 1. 317928 & -0.625760 & -3.273966 \\
\hline
\end{tabular}




\begin{tabular}{|c|c|c|c|}
\hline H48 & 0.950185 & 0.882357 & -2.983332 \\
\hline H49 & -6.416109 & 0.004335 & -1.861318 \\
\hline H50 & 6. 351542 & 0.236807 & 1. 957566 \\
\hline H51 & 0.779864 & -4.955826 & -1.789249 \\
\hline H52 & 3. 081779 & -5.507538 & -0.944447 \\
\hline H53 & 4. 469601 & -3.695467 & 0.045368 \\
\hline H54 & -0.039196 & -2.585496 & -1.566286 \\
\hline H55 & 2. 705415 & 5.840209 & -0.545581 \\
\hline H56 & 3. 994085 & 4. 078194 & 0.629090 \\
\hline H57 & -3.197592 & -5.812269 & 0.378253 \\
\hline H58 & -0.881487 & 4. 993167 & 2. 129009 \\
\hline H59 & -3.107916 & 5. 591926 & 1. 130255 \\
\hline $\mathrm{H} 60$ & -4.349171 & -3.875418 & -0.635620 \\
\hline H61 & -4.441399 & 3. 824083 & 0.004601 \\
\hline H62 & -0.075760 & 2. 617227 & 1. 909846 \\
\hline N63 & -0.234967 & -2.947344 & 2. 408961 \\
\hline C64 & -1.030659 & -5.626283 & 2. 063538 \\
\hline H65 & -1.361448 & -6.654168 & 1. 941601 \\
\hline C66 & 0.016161 & -5.307119 & 2. 897009 \\
\hline H67 & 0.549909 & -6.066858 & 3. 457742 \\
\hline C68 & 0.361394 & -3.944561 & 3. 050592 \\
\hline H69 & 1. 147076 & -3.661160 & 3. 747706 \\
\hline $\mathrm{C} 70$ & -0.427851 & 3. 444841 & -3.341802 \\
\hline H71 & -1.096822 & 3. 033073 & -4.093702 \\
\hline N72 & 0.176271 & 2. 566069 & -2.553384 \\
\hline C73 & -0.226921 & 4. 845181 & -3.268177 \\
\hline H74 & -0.764288 & 5. 502471 & -3.943291 \\
\hline C75 & 0.691788 & 5. 334197 & -2.368266 \\
\hline H76 & 0.915188 & 6. 395951 & -2.310630 \\
\hline 077 & -0.337270 & -0.592531 & 3. 814593 \\
\hline $\mathrm{H} 78$ & -1.049764 & -0.723383 & 4. 469477 \\
\hline H79 & -0.254005 & -1.448231 & 3. 288500 \\
\hline
\end{tabular}

Cartesian coordinates of product state associated with one water molecule approaching the parallel $\mathrm{Mn}^{\mathrm{IV}}$-oxyl radical in complex 2 :

Number of atoms in product-state complex: 79

$\begin{array}{lrrr}\text { Mn1 } & -0.951656 & 0.105843 & 0.780606 \\ \text { Mn2 } & 1.285573 & 0.133425 & -0.762715 \\ 03 & -0.434465 & 0.017291 & -1.026701 \\ 04 & 0.904533 & 0.052679 & 0.926001 \\ 05 & -1.257161 & 0.290971 & 2.613593 \\ 06 & 1.561051 & 0.493153 & -2.840780 \\ \text { N7 } & -1.862428 & -2.329499 & 1.212161\end{array}$




\begin{tabular}{|c|c|c|c|}
\hline N8 & -3.019524 & -0.252843 & -0.021899 \\
\hline N9 & -1.974564 & 2. 115559 & 0.666159 \\
\hline N10 & 1. 724694 & -1.833784 & -1.063711 \\
\hline N11 & 3. 267133 & 0.059817 & -0.275882 \\
\hline N12 & 1. 895469 & 2. 174430 & -0.709238 \\
\hline $\mathrm{C} 13$ & -2.838226 & -2.627345 & 0.351127 \\
\hline $\mathrm{C} 14$ & -3.443065 & -1.482342 & -0.384984 \\
\hline C15 & -4.468817 & -1.650309 & -1.321053 \\
\hline $\mathrm{C} 16$ & -5.078065 & -0.519758 & -1.867218 \\
\hline $\mathrm{C} 17$ & -4.674385 & 0.739383 & -1.443181 \\
\hline C18 & -3.634100 & 0.840438 & -0.505757 \\
\hline C19 & -3.170745 & 2. 140692 & 0.044988 \\
\hline $\mathrm{C} 20$ & 3. 003967 & -2.215207 & -0.779610 \\
\hline $\mathrm{C} 21$ & 3. 850373 & -1.144885 & -0.211468 \\
\hline C22 & 5. 117716 & -1.283988 & 0.362931 \\
\hline C23 & 5.731707 & -0.145593 & 0.889845 \\
\hline C24 & 5. 091146 & 1. 096028 & 0.833347 \\
\hline C25 & 3. 834097 & 1. 167838 & 0.227626 \\
\hline C26 & 3. 035891 & 2. 388649 & -0.028749 \\
\hline $\mathrm{C} 27$ & 0.867764 & -2.710773 & -1.610609 \\
\hline C28 & 3. 435838 & -3.512768 & -1.048593 \\
\hline C29 & 1. 245125 & -4.017859 & -1.912122 \\
\hline C30 & 2. 549095 & -4.423150 & -1.626667 \\
\hline C31 & 1. 245372 & 3. 232178 & -1.278648 \\
\hline C32 & 3. 474073 & 3. 687985 & 0. 302061 \\
\hline C33 & 1. 628541 & 4. 574541 & -0.976878 \\
\hline C34 & 2. 747408 & 4. 775588 & -0.135077 \\
\hline C35 & -1.444459 & -3.292392 & 2. 052878 \\
\hline С36 & -3.341276 & -3.954414 & 0.215047 \\
\hline C37 & -2.850554 & -4.957352 & 1. 014593 \\
\hline C38 & -1.881280 & -4.645353 & 2. 003879 \\
\hline C39 & -3.950403 & 3. 300654 & 0.009024 \\
\hline $\mathrm{C} 40$ & -1.529481 & 3. 216595 & 1. 283806 \\
\hline C41 & -2.247607 & 4. 412262 & 1. 293840 \\
\hline C42 & -3.478717 & 4. 452234 & 0.640736 \\
\hline H43 & -4.805789 & -2.633202 & -1.625092 \\
\hline H44 & -5.148612 & 1.627820 & -1.843033 \\
\hline H45 & 5. 613512 & -2.246763 & 0.413652 \\
\hline H46 & 5. 572908 & 1. 975347 & 1. 244677 \\
\hline H47 & 1. 199106 & -0.181217 & -3.440987 \\
\hline H48 & 0.964028 & 1. 285663 & -2.918624 \\
\hline H49 & -5.870168 & -0.626370 & -2.601580 \\
\hline H50 & 6. 712917 & -0.224693 & 1. 347274 \\
\hline H51 & 0.532907 & -4.693687 & -2.373084 \\
\hline
\end{tabular}




\begin{tabular}{|c|c|c|c|}
\hline H52 & 2. 879813 & -5.430422 & -1.860764 \\
\hline H53 & 4. 458483 & -3.804305 & -0.836216 \\
\hline H54 & -0.127777 & -2.326906 & -1.805122 \\
\hline H55 & 3. 060796 & 5. 785323 & 0.115538 \\
\hline H56 & 4. 381428 & 3. 830363 & 0.876909 \\
\hline H57 & -3.222660 & -5.973802 & 0.924533 \\
\hline H58 & -1.859462 & 5. 278429 & 1. 819161 \\
\hline H59 & -4.077845 & 5. 357644 & 0.641016 \\
\hline H60 & -4.123590 & -4.179853 & -0.497898 \\
\hline H61 & -4.926442 & 3. 304163 & -0.462569 \\
\hline H62 & -0.578132 & 3. 123000 & 1. 798761 \\
\hline N63 & -0.572437 & -2.926079 & 3. 048609 \\
\hline C64 & -1.367440 & -5.568750 & 2. 946147 \\
\hline H65 & -1.697735 & -6.603699 & 2. 914952 \\
\hline C66 & -0.469955 & -5.147774 & 3. 912222 \\
\hline H67 & -0.070850 & -5.829923 & 4. 654087 \\
\hline C68 & -0.105679 & -3.796902 & 3. 949129 \\
\hline H69 & 0.552187 & -3.391973 & 4. 711035 \\
\hline $\mathrm{C} 70$ & -0.341345 & 3. 937202 & -2.779923 \\
\hline H71 & -1.073315 & 3. 667076 & -3.537332 \\
\hline N72 & 0.291576 & 2. 930829 & -2.194421 \\
\hline C73 & -0.096128 & 5. 302945 & -2.493442 \\
\hline H74 & -0.662413 & 6.071193 & -3.008832 \\
\hline C75 & 0.900005 & 5. 622366 & -1.600076 \\
\hline H76 & 1. 157901 & 6. 656253 & -1.387797 \\
\hline 077 & -0.199110 & -0.270976 & 3. 447580 \\
\hline H78 & -0.467376 & 0.091848 & 4. 312690 \\
\hline H79 & -0.368648 & -1.896311 & 3. 179261 \\
\hline
\end{tabular}

Cartesian coordinates of the active $\left[\left(\mathrm{H}_{2} \mathrm{O}\right) \mathrm{Mn}^{\mathrm{IV}}(\mu-\mathrm{O})_{2} \mathrm{Mn}^{\mathrm{IV}} \mathrm{OO}\right]^{2+}$ species for complex 2 :

Number of atoms in $\left[\left(\mathrm{H}_{2} \mathrm{O}\right) \mathrm{Mn}^{\mathrm{IV}}(\mu-\mathrm{O})_{2} \mathrm{Mn}^{\mathrm{IV}} \mathrm{OO}\right]^{2+}$ species: 77

$\begin{array}{lrrr}\text { Mn1 } & 0.102492 & 0.012271 & 0.068468 \\ \text { Mn2 } & 0.035685 & -0.024723 & 2.749482 \\ 03 & 1.281682 & -0.084010 & 1.449046 \\ 04 & -1.130059 & 0.065009 & 1.347075 \\ 05 & -1.987729 & -0.419710 & -1.725576 \\ \text { 06 } & 1.626020 & 0.445643 & 4.096052 \\ \text { N7 } & 0.624147 & -2.050552 & -0.910326 \\ \text { N8 } & 1.505256 & 0.336924 & -1.510733 \\ \text { N9 } & -0.288836 & 2.045244 & -0.693249 \\ \text { N10 } & 0.135723 & -2.214791 & 3.381805 \\ \text { N11 } & -1.659871 & -0.388466 & 4.090230 \\ \text { N12 } & -0.722671 & 2.053507 & 3.803315\end{array}$




\begin{tabular}{|c|c|c|c|}
\hline C13 & 1. 777553 & -2.034004 & -1.578450 \\
\hline C14 & 2. 265125 & -0.679624 & -1.957511 \\
\hline C15 & 3. 356942 & -0.435387 & -2.796378 \\
\hline C16 & 3. 622031 & 0.876962 & -3.186878 \\
\hline $\mathrm{C} 17$ & 2. 779323 & 1. 907632 & -2.776069 \\
\hline C18 & 1. 706279 & 1. 598961 & -1.933416 \\
\hline C19 & 0.675371 & 2. 569730 & -1.485416 \\
\hline $\mathrm{C} 20$ & -0.906411 & -2.666434 & 4. 108333 \\
\hline $\mathrm{C} 21$ & -1.966940 & -1.662776 & 4. 391215 \\
\hline C22 & -3.210033 & -1.993022 & 4. 947725 \\
\hline C23 & -4.128032 & -0.977845 & 5. 192789 \\
\hline $\mathrm{C} 24$ & -3.783762 & 0.342988 & 4. 908748 \\
\hline C25 & -2.525922 & 0.608057 & 4. 360409 \\
\hline $\mathrm{C} 26$ & -2.005970 & 1. 987086 & 4. 138341 \\
\hline $\mathrm{C} 27$ & 1. 144267 & -3.041169 & 3. 085777 \\
\hline C28 & -0.947463 & -3.983429 & 4. 576991 \\
\hline C29 & 1. 179595 & -4.366497 & 3. 518567 \\
\hline C30 & 0.113171 & -4.839956 & 4. 281530 \\
\hline C31 & -0.095382 & 3. 254829 & 3. 774411 \\
\hline C32 & -2.786843 & 3. 153698 & 4. 355357 \\
\hline C33 & -0.798018 & 4. 476488 & 3. 991494 \\
\hline C34 & -2.186461 & 4. 388597 & 4. 261682 \\
\hline C35 & 0.020440 & -3.239425 & -0.637410 \\
\hline C36 & 2. 442481 & -3.220083 & -1.976216 \\
\hline C37 & 1. 857803 & -4.435826 & -1.703858 \\
\hline C38 & 0.609967 & -4.478888 & -1.039118 \\
\hline C39 & 0.640682 & 3. 910906 & -1.872935 \\
\hline C40 & -1.297089 & 2. 816911 & -0.270093 \\
\hline C41 & -1.408787 & 4. 158634 & -0.633291 \\
\hline C42 & -0.419528 & 4. 711434 & -1.445440 \\
\hline H43 & 3. 979397 & -1.243783 & -3.159578 \\
\hline H44 & 2. 953188 & 2. 921587 & -3.115753 \\
\hline H45 & -3.462674 & -3.021108 & 5. 177007 \\
\hline H46 & -4.479808 & 1. 142320 & 5. 130781 \\
\hline H47 & 2. 438115 & 0.084338 & 3. 700313 \\
\hline H48 & 1. 693770 & 1. 433070 & 3. 972825 \\
\hline H49 & 4. 465496 & 1. 090414 & -3.835883 \\
\hline H50 & -5.100085 & -1.209625 & 5. 616447 \\
\hline H51 & 2. 023758 & -5.001476 & 3. 271634 \\
\hline H52 & 0.106197 & -5.861059 & 4. 650406 \\
\hline H53 & -1.774287 & -4.336792 & 5. 182038 \\
\hline H54 & 1. 937911 & -2.613777 & 2. 479338 \\
\hline H55 & -2.761947 & 5. 296056 & 4. 423344 \\
\hline H56 & -3.840185 & 3. 081704 & 4. 597115 \\
\hline
\end{tabular}




\begin{tabular}{lrrr} 
H57 & 2.338274 & -5.361284 & -2.009791 \\
H58 & -2.252771 & 4.747835 & -0.291164 \\
H59 & -0.470983 & 5.752106 & -1.750460 \\
H60 & 3.390470 & -3.177180 & -2.498417 \\
H61 & 1.413765 & 4.328696 & -2.507354 \\
H62 & -2.018688 & 2.325371 & 0.375111 \\
N63 & -1.171469 & -3.166705 & 0.006209 \\
C64 & -0.102704 & -5.673137 & -0.757719 \\
H65 & 0.312488 & -6.631644 & -1.057137 \\
C66 & -1.317416 & -5.584172 & -0.121431 \\
H67 & -1.906258 & -6.467936 & 0.100832 \\
C68 & -1.804719 & -4.300193 & 0.241540 \\
H69 & -2.766333 & -4.215257 & 0.744525 \\
C70 & 1.914845 & 4.353927 & 3.568322 \\
H71 & 2.991906 & 4.286545 & 3.433498 \\
N72 & 1.252398 & 3.209323 & 3.579544 \\
C73 & 1.303742 & 5.621892 & 3.734917 \\
H74 & 1.914081 & 6.518223 & 3.711563 \\
C75 & -0.053703 & 5.683138 & 3.953117 \\
H76 & -0.556484 & 6.633119 & 4.113299 \\
077 & -3.113371 & -0.149257 & -1.360281 \\
\hline & & & \\
\hline
\end{tabular}

Cartesian coordinates of the active $\left[\left(\mathrm{H}_{2} \mathrm{O}\right) \mathrm{Mn}^{\mathrm{IV}}(\mu-\mathrm{O})_{2} \mathrm{Mn}^{\mathrm{V}} \mathrm{OO}\right]^{3+}$ species for complex 2 : Number of atoms in $\left[\left(\mathrm{H}_{2} \mathrm{O}\right) \mathrm{Mn}^{\mathrm{IV}}(\mu-\mathrm{O})_{2} \mathrm{Mn}^{\mathrm{V}} \mathrm{OO}\right]^{3+}$ species: 77

$\begin{array}{lrrr}\text { Mn1 } & -1.137099 & -0.112768 & 0.472754 \\ \text { Mn2 } & 1.254876 & 0.035293 & -0.776918 \\ \text { 03 } & -0.404012 & -0.224329 & -1.248657 \\ \text { 04 } & 0.614667 & 0.115249 & 0.863515 \\ \text { 05 } & -1.631533 & -0.303629 & 3.172799 \\ \text { 06 } & 1.809130 & 0.157167 & -2.794749 \\ \text { N7 } & -1.919293 & -2.217705 & 0.730539 \\ \text { N8 } & -3.135598 & -0.062833 & -0.050199 \\ \text { N9 } & -1.779732 & 1.896912 & 0.973711 \\ \text { N10 } & 1.820460 & -1.908704 & -0.761912 \\ \text { N11 } & 3.120883 & 0.160291 & 0.004523 \\ \text { N12 } & 1.725985 & 2.095465 & -0.905283 \\ \text { C13 } & -3.087508 & -2.434639 & 0.111246 \\ \text { C14 } & -3.772704 & -1.208210 & -0.369641 \\ \text { C15 } & -5.029162 & -1.166769 & -0.979110 \\ \text { C16 } & -5.625581 & 0.075672 & -1.203614 \\ \text { C17 } & -4.993855 & 1.242378 & -0.771868 \\ \text { C18 } & -3.733541 & 1.139810 & -0.176864 \\ \text { C19 } & -2.982860 & 2.252177 & 0.451382\end{array}$




\begin{tabular}{|c|c|c|c|}
\hline $\mathrm{C} 20$ & 3. 044985 & -2.172105 & -0.218613 \\
\hline C21 & 3. 741148 & -0.986666 & 0.319496 \\
\hline $\mathrm{C} 22$ & 4. 913136 & -0.963073 & 1. 082215 \\
\hline C23 & 5. 390930 & 0.272858 & 1. 522789 \\
\hline C24 & 4. 714187 & 1. 453181 & 1. 196933 \\
\hline C25 & 3. 559708 & 1. 362194 & 0.416726 \\
\hline C26 & 2. 755298 & 2. 479353 & -0.125993 \\
\hline $\mathrm{C} 27$ & 1. 102125 & -2.897924 & -1.317024 \\
\hline C28 & 3. 564527 & -3.464139 & -0.232334 \\
\hline C29 & 1. 577199 & -4.206890 & -1.372754 \\
\hline C30 & 2. 825611 & -4.493074 & -0.821380 \\
\hline C31 & 1. 111294 & 3. 013997 & -1.710234 \\
\hline C32 & 3. 088698 & 3. 838329 & 0.049004 \\
\hline C33 & 1. 393101 & 4. 407491 & -1.574577 \\
\hline C34 & 2. 381951 & 4. 800700 & -0.642683 \\
\hline C35 & -1.299546 & -3.244874 & 1. 380773 \\
\hline C36 & -3.681784 & -3.714470 & 0.040885 \\
\hline C37 & -3.074054 & -4.769839 & 0.687169 \\
\hline C38 & -1.872375 & -4.555663 & 1. 400650 \\
\hline C39 & -3.478233 & 3. 548150 & 0.598031 \\
\hline $\mathrm{C} 40$ & -1.059266 & 2. 799068 & 1. 655892 \\
\hline C41 & -1.504447 & 4. 103939 & 1. 862196 \\
\hline C42 & -2.731502 & 4. 483129 & 1. 318239 \\
\hline $\mathrm{H} 43$ & -5.550087 & -2.075166 & -1.256468 \\
\hline H44 & -5.484894 & 2. 201601 & -0.885771 \\
\hline H45 & 5. 437985 & -1.876126 & 1. 339357 \\
\hline H46 & 5. 092580 & 2. 409049 & 1. 540203 \\
\hline H47 & 1. 625984 & -0.624100 & -3.345759 \\
\hline H48 & 1. 172644 & 0.868586 & -3.082144 \\
\hline H49 & -6.600479 & 0.130541 & -1.677991 \\
\hline H50 & 6. 294768 & 0.318906 & 2. 122247 \\
\hline H51 & 0.978541 & -4.977350 & -1.846484 \\
\hline H52 & 3. 228678 & -5.500589 & -0.856502 \\
\hline H53 & 4. 545016 & -3.664432 & 0. 185382 \\
\hline H54 & 0.136218 & -2.610237 & -1.716601 \\
\hline H55 & 2. 619591 & 5. 853834 & -0.519162 \\
\hline H56 & 3. 907283 & 4. 121253 & 0.700278 \\
\hline H57 & -3.525716 & -5.758102 & 0.673187 \\
\hline H58 & -0.908567 & 4. 796291 & 2. 447191 \\
\hline H59 & -3.114376 & 5. 488731 & 1. 463147 \\
\hline H60 & -4.618717 & -3.863460 & -0.481836 \\
\hline H61 & -4.441906 & 3. 824997 & 0.186114 \\
\hline H62 & -0.111234 & 2. 447210 & 2. 050432 \\
\hline N63 & -0.145835 & -2.935138 & 2. 020395 \\
\hline
\end{tabular}




$\begin{array}{lrrr}\text { C64 } & -1.207017 & -5.556891 & 2.156795 \\ \text { H65 } & -1.620635 & -6.560407 & 2.204951 \\ \text { C66 } & -0.056319 & -5.222031 & 2.830048 \\ \text { H67 } & 0.474788 & -5.946448 & 3.438464 \\ \text { C68 } & 0.434647 & -3.894270 & 2.722052 \\ \text { H69 } & 1.346279 & -3.617604 & 3.248083 \\ \text { C70 } & -0.292299 & 3.387165 & -3.488922 \\ \text { H71 } & -0.906396 & 2.961296 & -4.278738 \\ \text { N72 } & 0.302289 & 2.520739 & -2.679428 \\ \text { C73 } & -0.145920 & 4.792836 & -3.389264 \\ \text { H74 } & -0.672387 & 5.439036 & -4.083409 \\ \text { C75 } & 0.709116 & 5.303091 & -2.439656 \\ \text { H76 } & 0.892188 & 6.371184 & -2.361063 \\ \text { 077 } & -0.847963 & 0.139777 & 3.986920\end{array}$

Cartesian coordinates of reactant state associated with oxygen releasing for complex $\mathbf{2}$ : Number of atoms in reactant-state complex: 80

\begin{tabular}{|c|c|c|c|}
\hline Mn1 & 0.097051 & -0.104122 & -0.043857 \\
\hline Mn2 & -0.000505 & -0.060352 & 2. 615732 \\
\hline 03 & 1. 266558 & -0.009592 & 1. 365394 \\
\hline 04 & -1.155906 & -0.201989 & 1. 193154 \\
\hline 05 & -1.325346 & -0.266373 & -1.522852 \\
\hline 06 & 1. 480639 & 0.525547 & 4. 048450 \\
\hline N7 & 0.717391 & -2.084721 & -0.728880 \\
\hline N8 & 1. 600054 & 0.260218 & -1.392065 \\
\hline N9 & -0.060546 & 1. 975338 & -0.405433 \\
\hline N10 & 0. 209330 & -2.205660 & 3. 200736 \\
\hline N11 & -1.662224 & -0.501380 & 3. 906330 \\
\hline N12 & -0.867660 & 1. 930633 & 3. 437682 \\
\hline $\mathrm{C} 13$ & 1. 794673 & -2.090757 & -1.524548 \\
\hline C14 & 2. 325141 & -0.762890 & -1.872569 \\
\hline C15 & 3. 452188 & -0.520737 & -2.657898 \\
\hline C16 & 3. 799885 & 0.797005 & -2.939480 \\
\hline $\mathrm{C} 17$ & 3. 021568 & 1. 841065 & -2.454151 \\
\hline C18 & 1. 906543 & 1. 538096 & -1.670956 \\
\hline C19 & 0.953749 & 2. 515086 & -1.124700 \\
\hline $\mathrm{C} 20$ & -0.851589 & -2.743427 & 3. 836650 \\
\hline $\mathrm{C} 21$ & -1.915678 & -1.788290 & 4. 203813 \\
\hline $\mathrm{C} 22$ & -3.112006 & -2.156436 & 4. 823917 \\
\hline $\mathrm{C} 23$ & -4.044717 & -1.171631 & 5. 123357 \\
\hline $\mathrm{C} 24$ & -3.768773 & 0.153868 & 4. 812317 \\
\hline C25 & -2.554722 & 0.461107 & 4. 198830 \\
\hline C26 & -2.118664 & 1. 834921 & 3. 878174 \\
\hline
\end{tabular}




\begin{tabular}{|c|c|c|c|}
\hline $\mathrm{C} 27$ & 1. 216898 & -2.993994 & 2. 819164 \\
\hline C28 & -0.908617 & -4.109937 & 4. 115732 \\
\hline $\mathrm{C} 29$ & 1. 234921 & -4.362476 & 3. 065012 \\
\hline C30 & 0.148956 & -4.925468 & 3. 728386 \\
\hline C31 & -0.317704 & 3. 151632 & 3. 240993 \\
\hline C32 & -2.939307 & 2. 971063 & 4. 062565 \\
\hline C33 & -1.071155 & 4. 348030 & 3. 414401 \\
\hline C34 & -2.420536 & 4. 217107 & 3. 815624 \\
\hline C35 & 0.103703 & -3.259407 & -0.418052 \\
\hline C36 & 2. 351776 & -3.283165 & -2.030877 \\
\hline C37 & 1. 760404 & -4.483184 & -1.722035 \\
\hline C38 & 0.608316 & -4.502170 & -0.910163 \\
\hline C39 & 1. 019681 & 3. 885993 & -1.357214 \\
\hline $\mathrm{C} 40$ & -1.044523 & 2. 755051 & 0.053368 \\
\hline C41 & -1.044559 & 4. 130991 & -0.161360 \\
\hline C42 & 0.009278 & 4. 704733 & -0.862996 \\
\hline H43 & 4. 050661 & -1.338190 & -3.048975 \\
\hline H44 & 3. 277573 & 2. 870387 & -2.687769 \\
\hline H45 & -3.318402 & -3.193421 & 5. 071898 \\
\hline H46 & -4.488243 & 0.931372 & 5. 050082 \\
\hline H47 & 2. 321444 & 0.081505 & 3. 852857 \\
\hline H48 & 1. 606717 & 1. 453650 & 3. 726929 \\
\hline H49 & 4. 674709 & 1. 009757 & -3.548517 \\
\hline H50 & -4.982994 & -1.436017 & 5. 604744 \\
\hline H51 & 2. 082665 & -4.966469 & 2. 752607 \\
\hline H52 & 0.125070 & -5.990310 & 3. 948794 \\
\hline H53 & -1.759691 & -4.538173 & 4. 638849 \\
\hline H54 & 2. 029375 & -2.492156 & 2. 291812 \\
\hline H55 & -3.033653 & 5. 107978 & 3. 942238 \\
\hline H56 & -3.972031 & 2. 862413 & 4. 379569 \\
\hline H57 & 2. 162844 & -5.417441 & -2.110293 \\
\hline H58 & -1.870787 & 4. 731836 & 0. 209799 \\
\hline H59 & 0.037181 & 5. 776423 & -1.046724 \\
\hline H60 & 3. 226652 & -3.251708 & -2.673186 \\
\hline H61 & 1. 836645 & 4. 309719 & -1.935550 \\
\hline H62 & -1.859706 & 2. 251492 & 0.581572 \\
\hline N63 & -0.996323 & -3.168308 & 0.361183 \\
\hline C64 & -0.092315 & -5.680052 & -0.562132 \\
\hline H65 & 0.261321 & -6.643061 & -0.926832 \\
\hline C66 & -1.215407 & -5.576404 & 0.219196 \\
\hline H67 & -1.793532 & -6.453086 & 0.498869 \\
\hline C68 & -1.621699 & -4.292293 & 0.650411 \\
\hline H69 & -2.515775 & -4.189880 & 1. 268649 \\
\hline $\mathrm{C} 70$ & 1. 572448 & 4. 319911 & 2. 693466 \\
\hline
\end{tabular}




$\begin{array}{lrrr}\text { H71 } & 2.631434 & 4.293039 & 2.436266 \\ \mathrm{~N} 72 & 0.997619 & 3.146689 & 2.900472 \\ \mathrm{C} 73 & 0.908944 & 5.560809 & 2.804393 \\ \mathrm{H} 74 & 1.455043 & 6.482481 & 2.622261 \\ \mathrm{C} 75 & -0.414289 & 5.575861 & 3.178002 \\ \mathrm{H} 76 & -0.955292 & 6.512786 & 3.304813 \\ \text { 077 } & -0.969321 & -0.240128 & -2.727822 \\ \text { 078 } & -3.567594 & 1.146946 & 1.408943 \\ \mathrm{H} 79 & -4.279339 & 0.971271 & 0.778816 \\ \mathrm{H} 80 & -2.893115 & 0.464391 & 1.221648\end{array}$

Cartesian coordinates of transition state associated with oxygen releasing for complex 2: Number of atoms in transition-state complex: 80

\begin{tabular}{|c|c|c|c|}
\hline Mn1 & 0.030818 & -0.028509 & 0.083626 \\
\hline Mn2 & 0.015553 & -0.088677 & 2. 743680 \\
\hline 03 & 1. 247074 & -0.071459 & 1. 452654 \\
\hline 04 & -1.185781 & -0.146262 & 1. 376912 \\
\hline 05 & -1.101710 & -0.161507 & -2.362106 \\
\hline 06 & 1. 571794 & 0.475399 & 4. 112589 \\
\hline N7 & 0.578995 & -1.994548 & -0.777122 \\
\hline N8 & 1.544375 & 0.347078 & -1.277762 \\
\hline N9 & -0.215483 & 2. 029212 & -0.415546 \\
\hline N10 & 0.163157 & -2.261272 & 3. 259512 \\
\hline N11 & -1.580449 & -0.501050 & 4. 141211 \\
\hline N12 & -0.780643 & 1. 924520 & 3. 606569 \\
\hline $\mathrm{C} 13$ & 1. 769645 & -2.003390 & -1.387354 \\
\hline $\mathrm{C} 14$ & 2. 292217 & -0.670047 & -1.735773 \\
\hline $\mathrm{C} 15$ & 3. 407821 & -0.421664 & -2.535134 \\
\hline C16 & 3. 713630 & 0.896430 & -2.862436 \\
\hline $\mathrm{C} 17$ & 2. 901240 & 1. 931771 & -2.415013 \\
\hline $\mathrm{C} 18$ & 1. 800840 & 1. 621555 & -1.613179 \\
\hline C19 & 0.807638 & 2. 584887 & -1.110177 \\
\hline $\mathrm{C} 20$ & -0.856278 & -2.770084 & 3. 981520 \\
\hline C21 & -1.827511 & -1.777050 & 4. 484960 \\
\hline $\mathrm{C} 22$ & -2.945363 & -2.109116 & 5. 256504 \\
\hline $\mathrm{C} 23$ & -3.811813 & -1.101056 & 5. 654881 \\
\hline C24 & -3.551295 & 0.213234 & 5. 284055 \\
\hline $\mathrm{C} 25$ & -2.414962 & 0.483972 & 4. 521893 \\
\hline C26 & -2.003228 & 1. 845705 & 4. 123656 \\
\hline $\mathrm{C} 27$ & 1. 072524 & -3.085604 & 2. 734133 \\
\hline $\mathrm{C} 28$ & -0.967025 & -4.142989 & 4. 207523 \\
\hline C29 & 1. 032324 & -4.463257 & 2. 919330 \\
\hline C30 & -0.007558 & -4.996527 & 3. 674219 \\
\hline
\end{tabular}




\begin{tabular}{|c|c|c|c|}
\hline C31 & -0.252044 & 3. 133745 & 3. 312897 \\
\hline C32 & -2.814916 & 2. 989009 & 4. 308618 \\
\hline C33 & 0.9967070 & 4. 3374720 & 3. 4796750 \\
\hline C34 & -2.315632 & 4. 224602 & 3. 973407 \\
\hline C35 & -0.048689 & -3.167188 & -0.505102 \\
\hline C36 & 2. 437626 & -3.202068 & -1.715429 \\
\hline C37 & 1. 836450 & -4.403163 & -1.426429 \\
\hline C38 & 0.560553 & -4.418658 & -0.823600 \\
\hline C39 & 0.856322 & 3. 956022 & -1.348968 \\
\hline $\mathrm{C} 40$ & -1.207100 & 2. 801137 & 0.039249 \\
\hline C41 & -1.223864 & 4. 176220 & -0.172084 \\
\hline C42 & -0.173735 & 4. 760454 & -0.872657 \\
\hline H43 & 4. 018349 & -1.234369 & -2.918088 \\
\hline H44 & 3. 116779 & 2. 958428 & -2.697040 \\
\hline H45 & -3.139029 & -3.137808 & 5.545708 \\
\hline H46 & -4.214394 & 1. 011652 & 5. 603974 \\
\hline H47 & 2. 390601 & 0.016250 & 3. 864600 \\
\hline H48 & 1. 694293 & 1. 396067 & 3. 776562 \\
\hline H49 & 4. 575342 & 1. 114633 & -3.488086 \\
\hline H50 & -4.684997 & -1.337086 & 6. 257687 \\
\hline H51 & 1. 801245 & -5.098554 & 2. 487002 \\
\hline H52 & -0.073791 & -6.068080 & 3. 848427 \\
\hline H53 & -1.786653 & -4.549001 & 4. 794220 \\
\hline H54 & 1. 852413 & -2.602494 & 2. 142830 \\
\hline H55 & -2.921282 & 5. 120131 & 4. 103464 \\
\hline H56 & -3.822128 & 2. 897285 & 4. 704865 \\
\hline H57 & 2. 328421 & -5.343107 & -1.672556 \\
\hline H58 & -2.057106 & 4. 770087 & 0.194667 \\
\hline H59 & -0.160385 & 5. 831671 & -1.061038 \\
\hline H60 & 3. 413007 & -3.176544 & -2.193095 \\
\hline H61 & 1. 678859 & 4. 392460 & -1.909888 \\
\hline H62 & -2.008758 & 2. 273055 & 0.552062 \\
\hline N63 & -1.272217 & -3.060251 & 0.062959 \\
\hline C64 & -0.159784 & -5.595391 & -0.518268 \\
\hline H65 & 0.270234 & -6.567914 & -0.753687 \\
\hline C66 & -1.398826 & -5.480271 & 0.062140 \\
\hline H67 & -1.993458 & -6.356763 & 0.305371 \\
\hline C68 & -1.906812 & -4.186903 & 0.327562 \\
\hline H69 & -2.894789 & -4.079798 & 0.778258 \\
\hline C70 & 1. 597209 & 4. 273165 & 2. 599705 \\
\hline H71 & 2. 638657 & 4. 235289 & 2. 279331 \\
\hline N72 & 1. 038245 & 3. 110456 & 2. 888456 \\
\hline $\mathrm{C} 73$ & 0.940507 & 5. 520070 & 2. 701116 \\
\hline H74 & 1. 474787 & 6. 431701 & 2. 447317 \\
\hline
\end{tabular}




$\begin{array}{lrrr}\text { C75 } & -0.357385 & 5.554186 & 3.151622 \\ \text { H76 } & -0.890793 & 6.496481 & 3.270349 \\ 077 & -0.397052 & -0.185564 & -3.364602 \\ 078 & -3.069495 & 0.410142 & -0.455332 \\ \text { H79 } & -3.508953 & -0.153936 & -1.105075 \\ \text { H80 } & -2.816867 & -0.183707 & 0.270133\end{array}$

Cartesian coordinates of product state associated with oxygen releasing for complex 2:

Number of atoms in product-state complex: 80

\begin{tabular}{|c|c|c|c|}
\hline Mn1 & -0.226506 & -0.100005 & 0.118775 \\
\hline Mn2 & -0.108090 & -0.008898 & 2. 766122 \\
\hline 03 & 1. 054895 & -0.069018 & 1. 385604 \\
\hline 04 & -1.390143 & -0.038503 & 1.501768 \\
\hline 05 & -0.678143 & 0.122117 & -3.700143 \\
\hline 06 & 1. 568740 & 0.454586 & 4. 017863 \\
\hline N7 & 0.457293 & -2.092195 & -0.874354 \\
\hline N8 & 1. 317234 & 0.321843 & -1.354328 \\
\hline N9 & -0.349157 & 2. 101660 & -0.369035 \\
\hline N10 & 0.013666 & -2.215031 & 3. 247112 \\
\hline N11 & -1.624261 & -0.423986 & 4. 265473 \\
\hline N12 & -0.786578 & 1. 988622 & 3. 739261 \\
\hline $\mathrm{C} 13$ & 1. 665645 & -2.032775 & -1.428585 \\
\hline $\mathrm{C} 14$ & 2. 112265 & -0.671598 & -1.788446 \\
\hline $\mathrm{C} 15$ & 3. 235901 & -0.409719 & -2.572688 \\
\hline $\mathrm{C} 16$ & 3. 523470 & 0.907887 & -2.910766 \\
\hline $\mathrm{C} 17$ & 2. 690859 & 1. 924704 & -2.465365 \\
\hline C18 & 1. 584149 & 1. 598548 & -1.675013 \\
\hline C19 & 0.637523 & 2. 599515 & -1.140696 \\
\hline $\mathrm{C} 20$ & -0.968287 & -2.707877 & 4. 028103 \\
\hline C21 & -1.891335 & -1.699910 & 4. 590821 \\
\hline C22 & -2.984359 & -2.020660 & 5. 402331 \\
\hline $\mathrm{C} 23$ & -3.804169 & -1.000167 & 5.862443 \\
\hline $\mathrm{C} 24$ & -3.521919 & 0.315552 & 5. 512703 \\
\hline C25 & -2.413061 & 0.572586 & 4. 706401 \\
\hline C26 & -1.983356 & 1.931413 & 4. 316558 \\
\hline $\mathrm{C} 27$ & 0.891067 & -3.050621 & 2. 687989 \\
\hline C28 & -1.081735 & -4.078323 & 4. 269071 \\
\hline C29 & 0.853137 & -4.425850 & 2. 892682 \\
\hline C30 & -0.155082 & -4.944135 & 3. 699174 \\
\hline C31 & -0.243568 & 3. 189418 & 3. 435711 \\
\hline C32 & -2.753555 & 3. 091793 & 4. 562774 \\
\hline C33 & -0.949029 & 4. 407729 & 3. 654955 \\
\hline C34 & -2.241797 & 4. 319805 & 4. 218579 \\
\hline
\end{tabular}




\begin{tabular}{|c|c|c|c|}
\hline C35 & -0.090434 & -3.296337 & -0.589047 \\
\hline C36 & 2. 434910 & -3.192875 & -1.679350 \\
\hline C37 & 1. 914322 & -4.423593 & -1.360238 \\
\hline C38 & 0.614779 & -4.513939 & -0.813240 \\
\hline C39 & 0.732631 & 3. 968162 & -1.399646 \\
\hline $\mathrm{C} 40$ & -1.245400 & 2. 931154 & 0.168557 \\
\hline C41 & -1.222977 & 4. 304513 & -0.050158 \\
\hline C42 & -0.213324 & 4. 827376 & -0.851893 \\
\hline H43 & 3. 867728 & -1.215339 & -2.935214 \\
\hline H44 & 2. 903651 & 2. 956050 & -2.730623 \\
\hline H45 & -3.196434 & -3.050882 & 5. 672341 \\
\hline H46 & -4.147419 & 1. 123709 & 5. 880590 \\
\hline H47 & 2. 343805 & -0.008375 & 3. 660127 \\
\hline H48 & 1. 684190 & 1. 390585 & 3. 723123 \\
\hline H49 & 4. 388218 & 1. 139306 & -3.527341 \\
\hline H50 & -4.657745 & -1.227746 & 6. 495876 \\
\hline H51 & 1. 599990 & -5.070911 & 2. 436755 \\
\hline H52 & -0.219243 & -6.013199 & 3. 889288 \\
\hline H53 & -1.871972 & -4.472352 & 4. 902364 \\
\hline H54 & 1. 643157 & -2.578696 & 2. 052257 \\
\hline H55 & -2.815973 & 5. 228045 & 4. 395309 \\
\hline H56 & -3.739458 & 3. 018515 & 5. 012878 \\
\hline H57 & 2. 487736 & -5.331443 & -1.541342 \\
\hline H58 & -1.982538 & 4. 944420 & 0. 391919 \\
\hline H59 & -0.162612 & 5. 894774 & -1.054958 \\
\hline H60 & 3. 427780 & -3.116784 & -2.113240 \\
\hline H61 & 1. 524285 & 4. 365342 & -2.029263 \\
\hline H62 & -2.000033 & 2. 456348 & 0.799072 \\
\hline N63 & -1.358313 & -3.253793 & -0.103301 \\
\hline C64 & -0.040055 & -5.721863 & -0.483844 \\
\hline H65 & 0.464106 & -6.673506 & -0.647265 \\
\hline C66 & -1.314859 & -5.667741 & 0. 026866 \\
\hline H67 & -1.859391 & -6.571927 & 0.285069 \\
\hline C68 & -1.933419 & -4.408086 & 0. 187319 \\
\hline H69 & -2.955228 & -4.350348 & 0.562949 \\
\hline $\mathrm{C} 70$ & 1. 592713 & 4. 294267 & 2. 638318 \\
\hline H71 & 2. 613491 & 4. 235069 & 2. 259912 \\
\hline N72 & 1. 021107 & 3. 141617 & 2. 942079 \\
\hline $\mathrm{C} 73$ & 0.973171 & 5.554877 & 2. 790138 \\
\hline H74 & 1. 515180 & 6.457095 & 2. 519902 \\
\hline C75 & -0.298545 & 5. 613163 & 3. 308233 \\
\hline H76 & -0.802174 & 6. 566024 & 3. 466069 \\
\hline 077 & 0.316871 & 0.091985 & -4.401289 \\
\hline 078 & -1.946570 & -0.566638 & -1.079964 \\
\hline
\end{tabular}




$\begin{array}{llll}\text { H79 } & -2.016643 & -1.518598 & -0.820402 \\ \text { H80 } & -2.691970 & -0.144984 & -0.621513\end{array}$

Cartesian coordinates of reactant state associated with oxygen releasing for complex 1:

Number of atoms in reactant-state complex: 70

\begin{tabular}{|c|c|c|c|}
\hline Mn1 & -1.357474 & -0.127792 & 0.500604 \\
\hline Mn2 & 1. 050646 & -0.049142 & -0.643365 \\
\hline 03 & -0.627330 & -0.000037 & -1.201197 \\
\hline 04 & 0.314820 & -0.266657 & 1. 052347 \\
\hline 05 & -2.006185 & -0.188322 & 2. 467482 \\
\hline 06 & 1. 660451 & 0.079149 & -2.739195 \\
\hline N7 & -1.908840 & -2.158318 & 0.494256 \\
\hline N8 & -3.268101 & -0.080492 & -0.178068 \\
\hline N9 & -1.876990 & 1. 932108 & 0.666496 \\
\hline N10 & 1. 822448 & -2.189119 & -0.788831 \\
\hline N11 & 3. 021857 & -0.025630 & 0.106407 \\
\hline N12 & 1. 666199 & 2. 102131 & -0.620919 \\
\hline $\mathrm{C} 13$ & -3.127148 & -2.428948 & -0.038118 \\
\hline C14 & -3.887615 & -1.241602 & -0.454628 \\
\hline C15 & -5.154677 & -1.239944 & -1.034529 \\
\hline $\mathrm{C} 16$ & -5.761165 & -0.016299 & -1.304437 \\
\hline $\mathrm{C} 17$ & -5.118793 & 1. 172347 & -0.97399 \\
\hline C18 & -3.852202 & 1. 110315 & -0.391439 \\
\hline C19 & -3.070220 & 2. 253702 & 0.100539 \\
\hline C20 & 2. 933105 & -2.407213 & -0.048953 \\
\hline C21 & 3. 626247 & -1.190465 & 0.418525 \\
\hline $\mathrm{C} 22$ & 4. 845887 & -1.203051 & 1. 093387 \\
\hline $\mathrm{C} 23$ & 5. 430885 & 0.008700 & 1. 444748 \\
\hline C24 & 4. 806420 & 1. 199717 & 1. 103258 \\
\hline C25 & 3. 589112 & 1. 154974 & 0.421546 \\
\hline C26 & 2. 854671 & 2. 349681 & -0.032430 \\
\hline $\mathrm{C} 27$ & 1. 168358 & -3.231888 & -1.309569 \\
\hline C28 & 3. 388876 & -3.701931 & 0.204428 \\
\hline C29 & 1. 576324 & -4.548053 & -1.123391 \\
\hline C30 & 2. 703422 & -4.782033 & -0.341158 \\
\hline C31 & 0.959168 & 3. 116767 & -1.123080 \\
\hline C32 & 3.346740 & 3. 648152 & 0.086465 \\
\hline C33 & 1. 388182 & 4. 437432 & -1.05339 \\
\hline C34 & 2. 602013 & 4. 703362 & -0.428259 \\
\hline C35 & -1.163717 & -3.147444 & 0.995016 \\
\hline C36 & -3.603085 & -3.735648 & -0.10499 \\
\hline C37 & -2.822360 & -4.767993 & 0.40559 \\
\hline C38 & -1.587140 & -4.472103 & 0.97325 \\
\hline
\end{tabular}




\begin{tabular}{|c|c|c|c|}
\hline C39 & -3.520832 & 3. 569840 & 0.072183 \\
\hline $\mathrm{C} 40$ & -1.138732 & 2.884673 & 1. 247207 \\
\hline C41 & -1.546157 & 4. 217289 & 1. 267179 \\
\hline C42 & -2.746010 & 4. 565406 & 0.660153 \\
\hline H43 & -5.666812 & -2.169543 & -1.266485 \\
\hline H44 & -5.603840 & 2. 126848 & -1.157134 \\
\hline H45 & 5. 342051 & -2.138080 & 1. 336205 \\
\hline H46 & 5. 258715 & 2. 149834 & 1. 370680 \\
\hline H47 & 2. 163749 & -0.664311 & -3.105549 \\
\hline H48 & 0.841046 & 0.128609 & -3.258349 \\
\hline H49 & -6.748430 & 0.010368 & -1.758666 \\
\hline H50 & 6. 378514 & 0.021970 & 1. 977545 \\
\hline H51 & 1. 024040 & -5.364471 & -1.581007 \\
\hline H52 & 3. 054238 & -5.795795 & -0.162818 \\
\hline H53 & 4. 272518 & -3.873661 & 0.812792 \\
\hline H54 & 0.279680 & -2.985590 & -1.892983 \\
\hline H55 & 0.785291 & 5. 233267 & -1.482717 \\
\hline H56 & 2. 974652 & 5. 721946 & -0.350404 \\
\hline H57 & 4. 303663 & 3. 841375 & 0.562863 \\
\hline H58 & 0.012306 & 2. 844878 & -1.591881 \\
\hline H59 & -3.185324 & -5.792368 & 0.372809 \\
\hline H60 & -0.960768 & -5.249501 & 1. 402208 \\
\hline H61 & -0.923333 & 4. 960194 & 1. 758239 \\
\hline H62 & -3.089503 & 5. 597025 & 0.656969 \\
\hline H63 & -4.580709 & -3.945127 & -0.531100 \\
\hline H64 & -0.208314 & -2.841119 & 1. 420754 \\
\hline H65 & -4.476317 & 3. 815780 & -0.383693 \\
\hline H66 & -0.201212 & 2. 563311 & 1. 712811 \\
\hline 067 & -3.237147 & -0.182203 & 2. 714242 \\
\hline 068 & 1. 538155 & 1. 716593 & 2. 590776 \\
\hline H69 & 1. 129182 & 0.887043 & 2. 272612 \\
\hline $\mathrm{H} 70$ & 1. 499724 & 1. 659707 & 3. 555183 \\
\hline
\end{tabular}

Cartesian coordinates of transition state associated with oxygen releasing for complex $\mathbf{1}$ :

Number of atoms in transition-state complex: 70

$\begin{array}{lrrr}\text { Mn1 } & -1.234311 & 0.003446 & 0.524453 \\ \text { Mn2 } & 1.156719 & 0.046502 & -0.658467 \\ 03 & -0.528547 & 0.197636 & -1.179701 \\ 04 & 0.463958 & -0.175047 & 1.034507 \\ 05 & -2.950647 & -0.113277 & 2.695727 \\ 06 & 1.760501 & 0.205094 & -2.755052 \\ \text { N7 } & -1.795177 & -2.037860 & 0.743660 \\ \text { N8 } & -3.168153 & -0.049534 & -0.169015\end{array}$




\begin{tabular}{|c|c|c|c|}
\hline N9 & -1.964188 & 2. 071431 & 0.716384 \\
\hline N10 & 1. 814606 & -2.140408 & -0.878842 \\
\hline N11 & 3. 181050 & -0.042125 & -0.041697 \\
\hline N12 & 1. 870646 & 2. 176444 & -0.597714 \\
\hline $\mathrm{C} 13$ & -2.955398 & -2.383042 & 0.128028 \\
\hline $\mathrm{C} 14$ & -3.743957 & -1.247805 & -0.375927 \\
\hline C15 & -5.007783 & -1.332626 & -0.958129 \\
\hline C16 & -5.662380 & -0.153663 & -1.301451 \\
\hline $\mathrm{C} 17$ & -5.072127 & 1. 076380 & -1.031663 \\
\hline C18 & -3.806468 & 1. 100560 & -0.443351 \\
\hline C19 & -3.095447 & 2. 308481 & 0.005457 \\
\hline $\mathrm{C} 20$ & 2. 957773 & -2.416575 & -0.212099 \\
\hline C21 & 3. 762183 & -1.239080 & 0.174272 \\
\hline C22 & 5. 055505 & -1.323862 & 0.691223 \\
\hline $\mathrm{C} 23$ & 5. 741348 & -0.150514 & 0.980680 \\
\hline C24 & 5. 138474 & 1. 076334 & 0.736313 \\
\hline C25 & 3. 844413 & 1. 103782 & 0.212944 \\
\hline C26 & 3. 121065 & 2. 347513 & -0.117714 \\
\hline $\mathrm{C} 27$ & 1. 047462 & -3.149393 & -1.301535 \\
\hline C28 & 3. 340308 & -3.731606 & 0. 057193 \\
\hline C29 & 1. 376014 & -4.485328 & -1.097054 \\
\hline C30 & 2. 541746 & -4.776376 & -0.395624 \\
\hline C31 & 1. 157826 & 3. 241770 & -0.968086 \\
\hline C32 & 3. 676221 & 3. 620688 & 0.014235 \\
\hline C33 & 1. 646110 & 4. 541076 & -0.876174 \\
\hline C34 & 2. 928057 & 4. 728442 & -0.370405 \\
\hline C35 & -1.033863 & -2.985757 & 1. 300879 \\
\hline C36 & -3.355297 & -3.714999 & 0.046550 \\
\hline C37 & -2.565951 & -4.697270 & 0.635330 \\
\hline C38 & -1.389812 & -4.329304 & 1. 282344 \\
\hline C39 & -3.564064 & 3. 602421 & -0.208134 \\
\hline $\mathrm{C} 40$ & -1.305302 & 3. 103301 & 1. 253095 \\
\hline C41 & -1.726637 & 4. 421108 & 1. 094556 \\
\hline $\mathrm{C} 42$ & -2.867459 & 4. 673849 & 0.342130 \\
\hline H43 & -5.484420 & -2.294319 & -1.125490 \\
\hline H44 & -5.602149 & 1. 997246 & -1.257439 \\
\hline H45 & 5. 532005 & -2.286417 & 0.850622 \\
\hline H46 & 5. 674034 & 1. 996677 & 0.948907 \\
\hline H47 & 2. 090519 & -0.615819 & -3.152486 \\
\hline H48 & 0.953840 & 0.427485 & -3.248720 \\
\hline H49 & -6.649363 & -0.194156 & -1.755305 \\
\hline H50 & 6. 750050 & -0.192755 & 1. 383855 \\
\hline H51 & 0.731000 & -5.273356 & -1.476986 \\
\hline H52 & 2. 832187 & -5.806655 & -0.204112 \\
\hline
\end{tabular}




$\begin{array}{lrrr}\text { H53 } & 4.249300 & -3.946400 & 0.612512 \\ \text { H54 } & 0.132311 & -2.859951 & -1.821395 \\ \text { H55 } & 1.037120 & 5.381538 & -1.198412 \\ \text { H56 } & 3.348811 & 5.727004 & -0.280613 \\ \text { H57 } & 4.681705 & 3.756557 & 0.402586 \\ \text { H58 } & 0.158470 & 3.027796 & -1.350102 \\ \text { H59 } & -2.873378 & -5.739410 & 0.595427 \\ \text { H60 } & -0.755773 & -5.067236 & 1.766456 \\ \text { H61 } & -1.169927 & 5.226949 & 1.565289 \\ \text { H62 } & -3.224684 & 5.690389 & 0.195245 \\ \text { H63 } & -4.278057 & -3.985000 & -0.460354 \\ \text { H64 } & -0.114665 & -2.629286 & 1.765615 \\ \text { H65 } & -4.467168 & 3.776504 & -0.787285 \\ \text { H66 } & -0.435288 & 2.842564 & 1.850243 \\ \text { 067 } & -3.929672 & -0.846734 & 2.650752 \\ \text { 068 } & -0.441615 & 1.047850 & 3.239058 \\ \text { H69 } & 0.281922 & 0.529895 & 2.849262 \\ \text { H70 } & -0.569255 & 0.700832 & 4.131774\end{array}$

Cartesian coordinates of product state associated with oxygen releasing for complex $\mathbf{1}$ :

Number of atoms in product-state complex: 70

$\begin{array}{lrrr}\text { Mn1 } & -1.060767 & -0.001660 & 0.640630 \\ \text { Mn2 } & 1.254624 & -0.034344 & -0.638572 \\ \text { 03 } & -0.483714 & -0.048207 & -1.082135 \\ \text { 04 } & 0.678911 & -0.057172 & 1.083164 \\ \text { 05 } & -4.353656 & 0.187354 & 2.883529 \\ \text { 06 } & 1.675730 & 0.006558 & -2.766028 \\ \text { N7 } & -1.834637 & -2.143652 & 0.798057 \\ \text { N8 } & -3.113433 & 0.021312 & 0.034791 \\ \text { N9 } & -1.754546 & 2.156759 & 0.748297 \\ \text { N10 } & 2.016999 & -2.194009 & -0.734294 \\ \text { N11 } & 3.320673 & 0.012279 & -0.105796 \\ \text { N12 } & 1.903805 & 2.139776 & -0.766389 \\ \text { C13 } & -3.000508 & -2.360899 & 0.151583 \\ \text { C14 } & -3.728999 & -1.141224 & -0.253484 \\ \text { C15 } & -4.990049 & -1.156289 & -0.851179 \\ \text { C16 } & -5.608688 & 0.052889 & -1.143988 \\ \text { C17 } & -4.974108 & 1.244968 & -0.820157 \\ \text { C18 } & -3.714280 & 1.199728 & -0.219461 \\ \text { C19 } & -2.970420 & 2.398809 & 0.217190 \\ \text { C20 } & 3.212609 & -2.377059 & -0.131875 \\ \text { C21 } & 3.962342 & -1.140326 & 0.170787 \\ \text { C22 } & 5.260567 & -1.133841 & 0.682030\end{array}$




\begin{tabular}{|c|c|c|c|}
\hline $\mathrm{C} 23$ & 5.888279 & 0.084946 & 0.907176 \\
\hline $\mathrm{C} 24$ & 5. 222518 & 1. 265280 & 0.605465 \\
\hline $\mathrm{C} 25$ & 3. 926699 & 1. 200379 & 0.088752 \\
\hline C26 & 3. 145423 & 2. 391262 & -0.303880 \\
\hline $\mathrm{C} 27$ & 1. 285886 & -3.259844 & -1.072423 \\
\hline $\mathrm{C} 28$ & 3. 691085 & -3.657481 & 0.152142 \\
\hline C29 & 1. 703071 & -4.565970 & -0.839973 \\
\hline C30 & 2. 928348 & -4.762449 & -0.210794 \\
\hline C31 & 1. 150647 & 3. 150119 & -1.203100 \\
\hline C32 & 3. 647793 & 3. 692314 & -0.248797 \\
\hline C33 & 1. 583942 & 4. 472467 & -1.191623 \\
\hline C34 & 2. 855095 & 4. 743459 & -0.697410 \\
\hline C35 & -1.128610 & -3.186754 & 1. 242095 \\
\hline C36 & -3.472451 & -3.655215 & -0.073362 \\
\hline C37 & -2.735818 & -4.736807 & 0.398238 \\
\hline C38 & -1.543106 & -4.503870 & 1. 075837 \\
\hline C39 & -3.483588 & 3. 694489 & 0.143255 \\
\hline $\mathrm{C} 40$ & -1.038736 & 3. 170546 & 1. 236386 \\
\hline C41 & -1.485247 & 4. 488220 & 1. 209754 \\
\hline C42 & -2.728826 & 4. 749992 & 0.644627 \\
\hline H43 & -5.491560 & -2.092662 & -1.077487 \\
\hline H44 & -5.457162 & 2. 194341 & -1.031783 \\
\hline H45 & 5. 782869 & -2.061760 & 0.894997 \\
\hline H46 & 5. 710109 & 2. 221581 & 0.768377 \\
\hline H47 & 2. 142143 & -0.762444 & -3.128960 \\
\hline H48 & 0.799526 & 0.008595 & -3.186790 \\
\hline H49 & -6.589918 & 0.065655 & -1.611936 \\
\hline H50 & 6. 898722 & 0.113948 & 1. 307168 \\
\hline H51 & 1. 082671 & -5.403984 & -1.146803 \\
\hline H52 & 3. 292452 & -5.766458 & -0.005857 \\
\hline H53 & 4. 647419 & -3.801209 & 0.647113 \\
\hline H54 & 0.325529 & -3.042248 & -1.543919 \\
\hline H55 & 0.940685 & 5. 264640 & -1.565819 \\
\hline H56 & 3. 234392 & 5. 762187 & -0.669168 \\
\hline H57 & 4. 648134 & 3. 892003 & 0.125190 \\
\hline H58 & 0.160676 & 2. 874326 & -1.569230 \\
\hline H59 & -3.096809 & -5.750658 & 0.241937 \\
\hline H60 & -0.946316 & -5.322189 & 1. 470416 \\
\hline H61 & -0.873115 & 5. 283974 & 1. 626062 \\
\hline H62 & -3.116927 & 5. 764954 & 0.602740 \\
\hline H63 & -4.405965 & -3.826712 & -0.602230 \\
\hline H64 & -0.191251 & -2.940149 & 1. 744387 \\
\hline H65 & -4.464298 & 3. 885600 & -0.283664 \\
\hline H66 & -0.068904 & 2. 901919 & 1. 657425 \\
\hline
\end{tabular}




$\begin{array}{lrrr}067 & -5.406128 & -0.227275 & 2.435327 \\ 068 & -1.351835 & 0.102303 & 2.788725 \\ \text { H69 } & -0.460670 & -0.076141 & 3.133102 \\ \text { H70 } & -1.948450 & -0.526371 & 3.222642\end{array}$

\section{Mulliken Atomic Charge and Spin Population Data for Complex 2 (and Some}

\section{Related Species)}

\section{Complex 2:}

\begin{tabular}{|c|c|c|c|c|c|}
\hline Atom & Mn1 & Mn2 & 03 & 04 & 05 \\
\hline Charge & 1. 058092 & 1. 179527 & -0.737064 & -0.729933 & -0.772705 \\
\hline Atom & 06 & N7 & N8 & N9 & N10 \\
\hline Charge & -0.744363 & -0.541425 & -0.566962 & -0.513857 & -0.541058 \\
\hline Atom & N11 & N12 & $\mathrm{C} 13$ & C14 & C15 \\
\hline Charge & -0.533713 & -0.581525 & 0.273687 & 0.303213 & -0.154599 \\
\hline Atom & C16 & $\mathrm{C} 17$ & $\mathrm{C} 18$ & $\mathrm{C} 19$ & $\mathrm{C} 20$ \\
\hline Charge & -0.096235 & -0.155160 & 0.300261 & 0.290373 & 0.325772 \\
\hline Atom & C21 & $\mathrm{C} 22$ & $\mathrm{C} 23$ & $\mathrm{C} 24$ & $\mathrm{C} 25$ \\
\hline Charge & 0.303634 & -0.144101 & -0.100027 & -0.144257 & 0.302191 \\
\hline Atom & C26 & $\mathrm{C} 27$ & $\mathrm{C} 28$ & $\mathrm{C} 29$ & C30 \\
\hline Charge & 0.315579 & 0.035130 & -0.144636 & -0.128491 & -0.092643 \\
\hline Atom & C31 & C32 & C33 & C34 & C35 \\
\hline Charge & 0.399011 & -0.163248 & 0.147105 & -0.139250 & 0.388963 \\
\hline Atom & C36 & C37 & C38 & C39 & $\mathrm{C} 40$ \\
\hline Charge & -0.169977 & -0.146753 & 0.143715 & -0.153487 & 0.027973 \\
\hline Atom & C41 & $\mathrm{C} 42$ & H43 & H44 & H45 \\
\hline Charge & -0.130028 & -0.097704 & 0.205084 & 0.202911 & 0.216306 \\
\hline Atom & $\mathrm{H} 46$ & $\mathrm{H} 47$ & $\mathrm{H} 48$ & H49 & $\mathrm{H} 50$ \\
\hline Charge & 0.216717 & 0.473287 & 0.489985 & 0.492190 & 0.468297 \\
\hline Atom & H51 & H52 & H53 & H54 & H55 \\
\hline Charge & 0.223163 & 0.234145 & 0.213228 & 0.231394 & 0.215145 \\
\hline Atom & H56 & H57 & H58 & H59 & $\mathrm{H} 60$ \\
\hline Charge & 0.218194 & 0.225813 & 0.211812 & 0.216238 & 0.202638 \\
\hline Atom & H61 & H62 & H63 & H64 & N65 \\
\hline Charge & 0.218745 & 0.201707 & 0.200931 & 0.180261 & -0.482247 \\
\hline Atom & C66 & H67 & C68 & H69 & N70 \\
\hline Charge & -0.133808 & 0.214309 & -0.147420 & 0.215210 & 0.041894 \\
\hline Atom & H71 & $\mathrm{C} 72$ & H73 & N74 & C75 \\
\hline Charge & 0.204034 & 0.042814 & 0.209545 & -0.460905 & -0.142362 \\
\hline Atom & $\mathrm{H} 76$ & $\mathrm{C} 77$ & H78 & & \\
\hline Charge & 0.220475 & -0.130438 & 0.219684 & & \\
\hline
\end{tabular}

Sum of Mulliken atomic charges $=3.00000$ 


\section{Complex 2:}

\begin{tabular}{|c|c|c|c|c|c|}
\hline Atom & Mn1 & Mn2 & 03 & 04 & 05 \\
\hline Spin & 3. 874401 & -2.629038 & -0.220737 & -0.201554 & 0.00841 \\
\hline Atom & 06 & N7 & N8 & N9 & N10 \\
\hline Spin & 0.008952 & 0.043557 & -0.014396 & 0.056410 & 0.02849 \\
\hline Atom & N11 & N12 & $\mathrm{C} 13$ & C14 & $\mathrm{C} 15$ \\
\hline Spin & 0.031534 & 0.010080 & -0.001520 & 0.009074 & -0.005450 \\
\hline Atom & $\mathrm{C} 16$ & $\mathrm{C} 17$ & C18 & C19 & $\mathrm{C} 20$ \\
\hline Spin & 0.009991 & -0.005939 & 0.008592 & -0.000350 & 0.000409 \\
\hline Atom & $\mathrm{C} 21$ & $\mathrm{C} 22$ & $\mathrm{C} 23$ & $\mathrm{C} 24$ & C25 \\
\hline Spin & -0.006938 & 0.002736 & -0.008967 & 0.001980 & -0.007076 \\
\hline Atom & $\mathrm{C} 26$ & $\mathrm{C} 27$ & $\mathrm{C} 28$ & C29 & C30 \\
\hline Spin & 0.001153 & -0.006407 & 0.001761 & 0.004418 & -0.006738 \\
\hline Atom & C31 & C32 & C33 & C34 & C35 \\
\hline Spin & -0.002148 & 0.000258 & 0.000796 & -0.003315 & 0.000406 \\
\hline Atom & C36 & C37 & C38 & C39 & $\mathrm{C} 40$ \\
\hline Spin & 0.002338 & -0.000150 & 0.002729 & 0.002624 & -0.001846 \\
\hline Atom & C41 & $\mathrm{C} 42$ & H43 & H44 & H45 \\
\hline Spin & 0.002898 & 0.000879 & 0.000361 & 0.000226 & 0.000167 \\
\hline Atom & H46 & H47 & $\mathrm{H} 48$ & H49 & $\mathrm{H} 50$ \\
\hline Spin & 0.000035 & -0.001361 & -0.000800 & 0.000903 & 0.001991 \\
\hline Atom & H51 & H52 & H53 & $\mathrm{H} 54$ & H55 \\
\hline Spin & -0.000443 & 0.000375 & -0.000315 & 0.000337 & 0.000492 \\
\hline Atom & H56 & H57 & H58 & H59 & $\mathrm{H} 60$ \\
\hline Spin & 0.000755 & 0.000150 & 0.000266 & -0.000033 & 0.000799 \\
\hline Atom & H61 & H62 & H63 & $\mathrm{H} 64$ & N65 \\
\hline Spin & -0.000110 & 0.000480 & 0.000665 & 0.001851 & 0.000496 \\
\hline Atom & C66 & H67 & C68 & H69 & N70 \\
\hline Spin & -0.000114 & 0.000261 & 0.000582 & -0.000038 & -0.000254 \\
\hline Atom & H71 & $\mathrm{C} 72$ & $\mathrm{H} 73$ & N74 & C75 \\
\hline Spin & 0.000026 & -0.001319 & 0.000116 & 0.001318 & 0.000792 \\
\hline Atom & H76 & $\mathrm{C} 77$ & $\mathrm{H} 78$ & & \\
\hline Spin & -0.000021 & -0.001036 & 0.000078 & & \\
\hline
\end{tabular}

Sum of Mulliken atomic spin densities $=1.00000$

Transition state associated with one water molecule approaching the antiparallel $\mathrm{Mn}^{\mathrm{IV}}$-oxyl radical (TS1):

$\begin{array}{lccccc}\text { Atom } & \text { Mn1 } & \text { Mn2 } & 03 & 04 & 05 \\ \text { Charge } & 1.058092 & 1.064813 & -0.693550 & -0.802082 & -0.329207 \\ \text { Atom } & 06 & \text { N7 } & \text { N8 } & \text { N9 } & \text { N10 }\end{array}$




\begin{tabular}{|c|c|c|c|c|c|}
\hline Charge & -0.744268 & -0.548859 & -0.540457 & -0.513804 & -0.540294 \\
\hline Atom & N11 & N12 & $\mathrm{C} 13$ & $\mathrm{C} 14$ & $\mathrm{C} 15$ \\
\hline Charge & -0.524043 & -0.566026 & 0.301020 & 0.303434 & -0.146302 \\
\hline Atom & $\mathrm{C} 16$ & $\mathrm{C} 17$ & $\mathrm{C} 18$ & C19 & $\mathrm{C} 20$ \\
\hline Charge & -0.102972 & -0.143943 & 0.304654 & 0.323418 & 0.320536 \\
\hline Atom & $\mathrm{C} 21$ & C22 & $\mathrm{C} 23$ & $\mathrm{C} 24$ & $\mathrm{C} 25$ \\
\hline Charge & 0.292561 & -0.148746 & -0.101029 & -0.146702 & 0.294138 \\
\hline Atom & C26 & $\mathrm{C} 27$ & $\mathrm{C} 28$ & C29 & C30 \\
\hline Charge & 0.301476 & 0.030780 & -0.146824 & -0.131078 & -0.094669 \\
\hline Atom & C31 & C32 & C33 & C34 & C35 \\
\hline Charge & 0.389127 & -0.165977 & 0.146929 & -0.141588 & 0.438133 \\
\hline Atom & C36 & C37 & C38 & C39 & $\mathrm{C} 40$ \\
\hline Charge & -0.163337 & -0.142433 & 0.129800 & -0.148254 & 0.066426 \\
\hline Atom & C41 & C42 & H43 & H44 & H45 \\
\hline Charge & -0.135224 & -0.097566 & 0.210143 & 0.210123 & 0.212524 \\
\hline Atom & $\mathrm{H} 46$ & H47 & $\mathrm{H} 48$ & H49 & $\mathrm{H} 50$ \\
\hline Charge & 0.215387 & 0.471044 & 0.491782 & 0.228030 & 0. 232318 \\
\hline Atom & H51 & H52 & H53 & H54 & H55 \\
\hline Charge & 0.212874 & 0.230717 & 0.214162 & 0.216328 & 0.224470 \\
\hline Atom & H56 & H57 & H58 & H59 & $\mathrm{H} 60$ \\
\hline Charge & 0.209777 & 0.224170 & 0. 206969 & 0.223407 & 0.210439 \\
\hline Atom & H61 & H62 & N63 & C64 & H65 \\
\hline Charge & 0.207830 & 0. 192892 & -0.548333 & -0.126654 & 0.222090 \\
\hline Atom & C66 & H67 & C68 & H69 & $\mathrm{C} 70$ \\
\hline Charge & -0.144952 & 0.224656 & 0.067885 & 0.214199 & 0.040017 \\
\hline Atom & $\mathrm{H} 71$ & N72 & $\mathrm{C} 73$ & $\mathrm{H} 74$ & $\mathrm{C} 75$ \\
\hline Charge & 0.208439 & -0.459752 & -0.143256 & 0.219849 & -0.132977 \\
\hline Atom & H76 & 077 & $\mathrm{H} 78$ & H78 & \\
\hline Charge & 0.218956 & -0.532551 & 0.510503 & 0.478308 & \\
\hline
\end{tabular}

Transition state associated with one water molecule approaching the antiparallel $\mathrm{Mn}^{\mathrm{IV}}$-oxyl radical (TS1):

$\begin{array}{lccccc}\text { Atom } & \text { Mn1 } & \text { Mn2 } & 03 & 04 & 05 \\ \text { Spin } & -2.146242 & 2.769139 & 0.209573 & 0.015774 & -0.011709 \\ \text { Atom } & 06 & \mathrm{~N} 7 & \mathrm{~N} 8 & \mathrm{~N} 9 & \mathrm{~N} 10 \\ \text { Spin } & -0.009540 & 0.007267 & 0.018159 & 0.026808 & -0.038338 \\ \text { Atom } & \mathrm{N} 11 & \mathrm{~N} 12 & \mathrm{C} 13 & \mathrm{C} 14 & \mathrm{C} 15 \\ \text { Spin } & -0.022939 & 0.000998 & -0.000395 & 0.008614 & -0.006125 \\ \text { Atom } & \mathrm{C} 16 & \mathrm{C} 17 & \mathrm{C} 18 & \mathrm{C} 19 & \mathrm{C} 20 \\ \text { Spin } & 0.009500 & -0.005354 & 0.007046 & 0.003236 & 0.002469 \\ \text { Atom } & \mathrm{C} 21 & \mathrm{C} 22 & \mathrm{C} 23 & \mathrm{C} 24 & \mathrm{C} 25\end{array}$




\begin{tabular}{|c|c|c|c|c|c|}
\hline Spin & 0.006345 & -0.002336 & 0.008957 & -0.002276 & 0.007841 \\
\hline Atom & $\mathrm{C} 26$ & $\mathrm{C} 27$ & $\mathrm{C} 28$ & C29 & C30 \\
\hline Spin & -0.001425 & 0.008808 & -0.004014 & -0.006543 & 0.009637 \\
\hline Atom & C31 & C32 & C33 & C34 & C35 \\
\hline Spin & 0.001957 & 0.000060 & 0.000161 & 0.002010 & -0.002036 \\
\hline Atom & С 36 & C37 & C38 & C39 & C40 \\
\hline Spin & 0.001640 & -0.001116 & 0.001138 & 0.000029 & 0.004725 \\
\hline Atom & $\mathrm{C} 41$ & $\mathrm{C} 42$ & $\mathrm{H} 43$ & H44 & H45 \\
\hline Spin & -0.001516 & 0.003512 & 0.000258 & 0.000296 & -0.000076 \\
\hline Atom & H46 & $\mathrm{H} 47$ & H48 & H49 & H50 \\
\hline Spin & 0.000080 & 0.001838 & 0.001101 & -0.000451 & -0.000387 \\
\hline Atom & $\mathrm{H} 51$ & H52 & H53 & H54 & H55 \\
\hline Spin & 0.000215 & -0.000478 & -0.000453 & -0.000649 & -0.000107 \\
\hline Atom & H56 & $\mathrm{H} 57$ & $\mathrm{H} 58$ & H59 & $\mathrm{H} 60$ \\
\hline Spin & -0.000131 & 0.000036 & -0.000025 & -0.000167 & 0.000199 \\
\hline Atom & H61 & H62 & N63 & C64 & H65 \\
\hline Spin & 0.000511 & 0.000669 & 0.000388 & -0.000689 & -0.000037 \\
\hline Atom & C66 & H67 & C68 & H69 & $\mathrm{C} 70$ \\
\hline Spin & 0.000890 & -0.000037 & 0.000132 & 0.000022 & 0.000894 \\
\hline Atom & $\mathrm{H} 71$ & N72 & $\mathrm{C} 73$ & $\mathrm{H} 74$ & C75 \\
\hline Spin & -0.000071 & -0.000733 & -0.000404 & 0.000006 & 0.000661 \\
\hline Atom & H76 & 077 & $\mathrm{H} 78$ & H78 & \\
\hline Spin & -0.000015 & 0.126184 & -0.000955 & -0.002012 & \\
\hline
\end{tabular}

Sum of Mulliken atomic spin densities $=1.00000$

Transition state associated with one water molecule approaching the parallel $\mathrm{Mn}^{\mathrm{IV}}$-oxyl radical (TS1):

$\begin{array}{lccccc}\text { Atom } & \text { Mn1 } & \text { Mn2 } & \text { 03 } & 04 & 05 \\ \text { Charge } & 1.132926 & 1.125115 & -0.712098 & -0.728056 & -0.374188 \\ \text { Atom } & 06 & \mathrm{~N} 7 & \mathrm{~N} 8 & \mathrm{~N} 9 & \mathrm{~N} 10 \\ \text { Charge } & -0.745136 & -0.525910 & -0.554281 & -0.528556 & -0.536243 \\ \text { Atom } & \mathrm{N} 11 & \mathrm{~N} 12 & \mathrm{C} 13 & \mathrm{C} 14 & \mathrm{C} 15 \\ \text { Charge } & -0.530156 & -0.578817 & 0.288822 & 0.305094 & -0.150694 \\ \text { Atom } & \mathrm{C} 16 & \mathrm{C} 17 & \mathrm{C} 18 & \mathrm{C} 19 & \mathrm{C} 20 \\ \text { Charge } & -0.097754 & -0.150782 & 0.304651 & 0.324337 & 0.323830 \\ \text { Atom } & \mathrm{C} 21 & \mathrm{C} 22 & \mathrm{C} 23 & \mathrm{C} 24 & \mathrm{C} 25 \\ \text { Charge } & 0.301932 & -0.145286 & -0.099861 & -0.143116 & 0.302819 \\ \text { Atom } & \mathrm{C} 26 & \mathrm{C} 27 & \mathrm{C} 28 & \mathrm{C} 29 & \mathrm{C} 30 \\ \text { Charge } & 0.313437 & 0.029493 & -0.147443 & -0.130756 & -0.095078 \\ \text { Atom } & \mathrm{C} 31 & \mathrm{C} 32 & \mathrm{C} 33 & \mathrm{C} 34 & \text { C35 } \\ \text { Charge } & 0.394947 & -0.163319 & 0.146855 & -0.140578 & 0.389387 \\ \text { Atom } & \mathrm{C} 36 & \mathrm{C} 37 & \mathrm{C} 38 & \mathrm{C} 39 & \text { C40 }\end{array}$




$\begin{array}{lccccc}\text { Charge } & -0.164997 & -0.144990 & 0.136221 & -0.152292 & 0.072649 \\ \text { Atom } & \text { C41 } & \text { C42 } & \text { H43 } & \text { H44 } & \text { H45 } \\ \text { Charge } & -0.137403 & -0.094973 & 0.209065 & 0.208345 & 0.213313 \\ \text { Atom } & \text { H46 } & \text { H47 } & \text { H48 } & \text { H49 } & \text { H50 } \\ \text { Charge } & 0.215857 & 0.471263 & 0.490213 & 0.227150 & 0.232591 \\ \text { Atom } & \text { H51 } & \text { H52 } & \text { H53 } & \text { H54 } & \text { H55 } \\ \text { Charge } & 0.211962 & 0.229539 & 0.213866 & 0.217259 & 0.224994 \\ \text { Atom } & \text { H56 } & \text { H57 } & \text { H58 } & \text { H59 } & \text { H60 } \\ \text { Charge } & 0.211528 & 0.219917 & 0.208429 & 0.223048 & 0.206067 \\ \text { Atom } & \text { H61 } & \text { H62 } & \text { N63 } & \text { C64 } & \text { H65 } \\ \text { Charge } & 0.206025 & 0.218576 & -0.496935 & -0.133412 & 0.217443 \\ \text { Atom } & \text { C66 } & \text { H67 } & \text { C68 } & \text { H69 } & \text { C70 } \\ \text { Charge } & -0.144342 & 0.217917 & 0.043251 & 0.205749 & 0.040503 \\ \text { Atom } & \text { H71 } & \mathrm{N} 72 & \mathrm{C} 73 & \mathrm{H} 74 & \mathrm{C} 75 \\ \text { Charge } & 0.209441 & -0.460567 & -0.142699 & 0.220129 & -0.132545 \\ \text { Atom } & \text { H76 } & 077 & \text { H78 } & \text { H78 } & \\ \text { Charge } & 0.219103 & -0.610110 & 0.469660 & 0.498650 & \end{array}$

Sum of Mulliken atomic charges $=2.00000$

Transition state associated with one water molecule approaching the parallel $\mathrm{Mn}^{\mathrm{IV}}$-oxyl radical (TS1):

$\begin{array}{lccccc}\text { Atom } & \text { Mn1 } & \text { Mn2 } & \text { O3 } & 04 & 05 \\ \text { Spin } & 3.294939 & -2.663473 & -0.212175 & -0.113478 & 0.452776 \\ \text { Atom } & 06 & \mathrm{~N} 7 & \mathrm{~N} 8 & \mathrm{~N} 9 & \mathrm{~N} 10 \\ \text { Spin } & 0.009886 & 0.011891 & -0.014071 & -0.045660 & 0.029044 \\ \text { Atom } & \mathrm{N} 11 & \mathrm{~N} 12 & \mathrm{C} 13 & \mathrm{C} 14 & \mathrm{C} 15 \\ \text { Spin } & 0.027325 & 0.010647 & -0.003273 & 0.009185 & -0.005106 \\ \text { Atom } & \mathrm{C} 16 & \mathrm{C} 17 & \mathrm{C} 18 & \mathrm{C} 19 & \mathrm{C} 20 \\ \text { Spin } & 0.009915 & -0.003345 & 0.004763 & 0.004689 & -0.000836 \\ \text { Atom } & \mathrm{C} 21 & \mathrm{C} 22 & \mathrm{C} 23 & \mathrm{C} 24 & \mathrm{C} 25 \\ \text { Spin } & -0.007778 & 0.002923 & -0.010462 & 0.002543 & -0.008369 \\ \text { Atom } & \mathrm{C} 26 & \mathrm{C} 27 & \mathrm{C} 28 & \mathrm{C} 29 & \mathrm{C} 30 \\ \text { Spin } & 0.000902 & -0.007937 & 0.002507 & 0.005417 & -0.008292 \\ \text { Atom } & \mathrm{C} 31 & \mathrm{C} 32 & \mathrm{C} 33 & \mathrm{C} 34 & \mathrm{C} 35 \\ \text { Spin } & -0.002322 & 0.000484 & 0.001036 & -0.003528 & -0.000483 \\ \text { Atom } & \mathrm{C} 36 & \mathrm{C} 37 & \mathrm{C} 38 & \mathrm{C} 39 & \mathrm{C} 40 \\ \text { Spin } & 0.001611 & -0.001378 & 0.001951 & -0.004469 & 0.010236 \\ \text { Atom } & \mathrm{C} 41 & \mathrm{C} 42 & \mathrm{H} 43 & \mathrm{H} 44 & \mathrm{H} 45 \\ \text { Spin } & -0.008082 & 0.012411 & 0.000310 & 0.000113 & 0.000114 \\ \text { Atom } & \mathrm{H} 46 & \mathrm{H} 47 & \mathrm{H} 48 & \mathrm{H} 49 & \mathrm{H} 50 \\ \text { Spin } & -0.000016 & -0.001664 & -0.001065 & -0.000422 & 0.000447 \\ \text { Atom } & \mathrm{H} 51 & \mathrm{H} 52 & \mathrm{H} 53 & \mathrm{H} 54 & \mathrm{H} 55\end{array}$




$\begin{array}{lccccc}\text { Spin } & -0.000344 & 0.000412 & 0.000459 & 0.000543 & 0.000168 \\ \text { Atom } & \text { H56 } & \text { H57 } & \text { H58 } & \text { H59 } & \text { H60 } \\ \text { Spin } & 0.000232 & 0.000047 & 0.000214 & -0.000675 & -0.000073 \\ \text { Atom } & \text { H61 } & \text { H62 } & \text { N63 } & \text { C64 } & \text { H65 } \\ \text { Spin } & -0.000467 & -0.001058 & 0.001081 & -0.000818 & 0.000120 \\ \text { Atom } & \text { C66 } & \text { H67 } & \text { C68 } & \text { H69 } & \text { C70 } \\ \text { Spin } & 0.000868 & -0.000030 & -0.000540 & 0.000053 & -0.001452 \\ \text { Atom } & \text { H71 } & \text { N72 } & \text { C73 } & \text { H74 } & \text { C75 } \\ \text { Spin } & 0.000129 & 0.001580 & 0.000853 & -0.000021 & -0.001094 \\ \text { Atom } & \text { H76 } & 077 & \text { H78 } & \text { H78 } & \\ \text { Spin } & 0.000095 & 0.230749 & -0.006530 & -0.004881 & \end{array}$

Sum of Mulliken atomic spin densities $=1.00000$

Transition state associated with oxygen releasing for complex $\mathbf{2}$ :

$\begin{array}{lccccc}\text { Atom } & \text { Mn1 } & \text { Mn2 } & \text { O3 } & \text { O4 } & \text { O5 } \\ \text { Charge } & 1.311390 & 1.128879 & -0.812085 & -0.831072 & -0.019053 \\ \text { Atom } & \text { 06 } & \text { N7 } & \text { N8 } & \text { N9 } & \text { N10 } \\ \text { Charge } & -0.825418 & -0.677298 & -0.685241 & -0.626535 & -0.598001 \\ \text { Atom } & \mathrm{N} 11 & \mathrm{~N} 12 & \mathrm{C} 13 & \mathrm{C} 14 & \mathrm{C} 15 \\ \text { Charge } & -0.673835 & -0.644504 & 0.349803 & 0.363961 & -0.219173 \\ \text { Atom } & \mathrm{C} 16 & \mathrm{C} 17 & \mathrm{C} 18 & \mathrm{C} 19 & \mathrm{C} 20 \\ \text { Charge } & -0.139803 & -0.220030 & 0.366333 & 0.355914 & 0.356014 \\ \text { Atom } & \mathrm{C} 21 & \mathrm{C} 22 & \mathrm{C} 23 & \mathrm{C} 24 & \mathrm{C} 25 \\ \text { Charge } & 0.364528 & -0.222562 & -0.137135 & -0.219167 & 0.364239 \\ \text { Atom } & \mathrm{C} 26 & \mathrm{C} 27 & \mathrm{C} 28 & \mathrm{C} 29 & \mathrm{C} 30 \\ \text { Charge } & 0.334039 & 0.063925 & -0.223624 & -0.201738 & -0.135558 \\ \text { Atom } & \mathrm{C} 31 & \mathrm{C} 32 & \mathrm{C} 33 & \mathrm{C} 34 & \mathrm{C} 35 \\ \text { Charge } & 0.508323 & -0.229215 & 0.094869 & -0.184928 & 0.490050 \\ \text { Atom } & \mathrm{C} 36 & \mathrm{C} 37 & \mathrm{C} 38 & \mathrm{C} 39 & \mathrm{C} 40 \\ \text { Charge } & -0.232001 & -0.183051 & 0.092862 & -0.223457 & 0.089828 \\ \text { Atom } & \mathrm{C} 41 & \mathrm{C} 42 & \mathrm{H} 43 & \mathrm{H} 44 & \mathrm{H} 45 \\ \text { Charge } & -0.205198 & -0.138078 & 0.230716 & 0.229994 & 0.225794 \\ \text { Atom } & \mathrm{H} 46 & \mathrm{H} 47 & \mathrm{H} 48 & \mathrm{H} 49 & \mathrm{H} 50 \\ \text { Charge } & 0.227745 & 0.486110 & 0.509925 & 0.251773 & 0.250331 \\ \text { Atom } & \mathrm{H} 51 & \mathrm{H} 52 & \mathrm{H} 53 & \mathrm{H} 54 & \mathrm{H} 55 \\ \text { Charge } & 0.222125 & 0.239088 & 0.218850 & 0.199581 & 0.241881 \\ \text { Atom } & \mathrm{H} 56 & \mathrm{H} 57 & \mathrm{H} 58 & \mathrm{H} 59 & \mathrm{H} 60 \\ \text { Charge } & 0.227225 & 0.241805 & 0.223533 & 0.238696 & 0.226641 \\ \text { Atom } & \mathrm{H} 61 & \mathrm{H} 62 & \mathrm{~N} 63 & \mathrm{C} 64 & \mathrm{H} 65 \\ \text { Charge } & 0.220623 & 0.231322 & -0.457249 & -0.181541 & 0.234942 \\ \text { Atom } & \mathrm{C} 66 & \mathrm{H} 67 & \mathrm{C} 68 & \mathrm{H} 69 & \mathrm{C} 70 \\ \text { Charge } & -0.209895 & 0.235724 & 0.034382 & 0.207362 & 0.044974\end{array}$




$\begin{array}{lccccc}\text { Atom } & \mathrm{H} 71 & \mathrm{~N} 72 & \mathrm{C} 73 & \mathrm{H} 74 & \mathrm{C} 75 \\ \text { Charge } & 0.217640 & -0.532789 & -0.217201 & 0.238199 & -0.176059 \\ \text { Atom } & \mathrm{H} 76 & 077 & 078 & \text { H79 } & \text { H80 } \\ \text { Charge } & 0.237925 & -0.011149 & -0.853576 & 0.447690 & 0.469668\end{array}$

Sum of Mulliken atomic charges $=2.00000$

Transition state associated with oxygen releasing for complex $\mathbf{2}$ :

\begin{tabular}{|c|c|c|c|c|c|}
\hline Atom & Mn1 & Mn2 & 03 & 04 & 05 \\
\hline Spin & -3.519949 & 3. 664865 & -0.064798 & -0.027321 & 0.940353 \\
\hline Atom & 06 & N7 & N8 & N9 & N10 \\
\hline Spin & -0.009368 & -0.009791 & 0.026115 & -0.007618 & 0.040445 \\
\hline Atom & N11 & N12 & $\mathrm{C} 13$ & $\mathrm{C} 14$ & $\mathrm{C} 15$ \\
\hline Spin & -0.010374 & 0.028078 & -0.011000 & -0.013283 & 0.008395 \\
\hline Atom & C16 & $\mathrm{C} 17$ & $\mathrm{C} 18$ & C19 & $\mathrm{C} 20$ \\
\hline Spin & -0.015466 & 0.008787 & -0.013770 & -0.006915 & 0.003402 \\
\hline Atom & $\mathrm{C} 21$ & $\mathrm{C} 22$ & $\mathrm{C} 23$ & $\mathrm{C} 24$ & $\mathrm{C} 25$ \\
\hline Spin & 0.009058 & -0.005776 & 0.010240 & -0.005554 & 0.009681 \\
\hline Atom & $\mathrm{C} 26$ & $\mathrm{C} 27$ & $\mathrm{C} 28$ & $\mathrm{C} 29$ & C30 \\
\hline Spin & 0.005264 & 0.001275 & 0.000559 & 0.000428 & 0.005610 \\
\hline Atom & C31 & C32 & C33 & C34 & C35 \\
\hline Spin & 0.003753 & -0.001545 & 0.001221 & 0.007188 & -0.006081 \\
\hline Atom & C36 & $\mathrm{C} 37$ & C38 & C39 & $\mathrm{C} 40$ \\
\hline Spin & 0.005014 & -0.014558 & 0. 001079 & 0.003494 & -0.012384 \\
\hline Atom & C41 & $\mathrm{C} 42$ & $\mathrm{H} 43$ & H44 & H45 \\
\hline Spin & 0.005188 & -0.015101 & -0.000399 & -0.000217 & 0.000221 \\
\hline Atom & $\mathrm{H} 46$ & $\mathrm{H} 47$ & $\mathrm{H} 48$ & H49 & H50 \\
\hline Spin & 0.000457 & 0.001757 & 0.000452 & 0.000903 & -0.000611 \\
\hline Atom & H51 & H52 & $\mathrm{H} 53$ & H54 & H55 \\
\hline Spin & 0.000938 & -0.000468 & 0.000716 & 0.002395 & -0.000505 \\
\hline Atom & H56 & $\mathrm{H} 57$ & H58 & H59 & $\mathrm{H} 60$ \\
\hline Spin & 0.000642 & 0.000949 & -0.000941 & 0.001048 & -0.000396 \\
\hline Atom & H61 & H62 & N63 & C64 & H65 \\
\hline Spin & -0.000324 & -0.000867 & 0.005042 & -0.002399 & -0.000013 \\
\hline Atom & C66 & H67 & C68 & H69 & $\mathrm{C} 70$ \\
\hline Spin & 0.001683 & -0.000040 & -0.003441 & 0.000287 & 0.000619 \\
\hline Atom & $\mathrm{H} 71$ & N72 & $\mathrm{C} 73$ & $\mathrm{H} 74$ & $\mathrm{C} 75$ \\
\hline Spin & 0.000018 & -0.001190 & -0.000238 & -0.000006 & 0.000618 \\
\hline Atom & H76 & 077 & 078 & H79 & $\mathrm{H} 80$ \\
\hline Spin & 0.000221 & 0.971673 & 0.001366 & -0.000235 & 0.001448 \\
\hline
\end{tabular}


Transition state associated with oxygen releasing for complex $\mathbf{1}$ :

$\begin{array}{lccccc}\text { Atom } & \text { Mn1 } & \text { Mn2 } & \text { O3 } & \text { O4 } & \text { O5 } \\ \text { Charge } & 1.300078 & 1.160362 & -0.818460 & -0.848644 & -0.007915 \\ \text { Atom } & 06 & \mathrm{~N} 7 & \mathrm{~N} 8 & \mathrm{~N} 9 & \mathrm{~N} 10 \\ \text { Charge } & -0.813125 & -0.638805 & -0.696180 & -0.607651 & -0.595400 \\ \text { Atom } & \mathrm{N} 11 & \mathrm{~N} 12 & \mathrm{C} 13 & \mathrm{C} 14 & \mathrm{C} 15 \\ \text { Charge } & -0.691216 & -0.602425 & 0.362354 & 0.368580 & -0.221388 \\ \text { Atom } & \mathrm{C} 16 & \mathrm{C} 17 & \mathrm{C} 18 & \mathrm{C} 19 & \mathrm{C} 20 \\ \text { Charge } & -0.139441 & -0.218220 & 0.363437 & 0.343392 & 0.331554 \\ \text { Atom } & \mathrm{C} 21 & \mathrm{C} 22 & \mathrm{C} 23 & \mathrm{C} 24 & \mathrm{C} 25 \\ \text { Charge } & 0.361363 & -0.222045 & -0.136899 & -0.220707 & 0.361784 \\ \text { Atom } & \mathrm{C} 26 & \mathrm{C} 27 & \mathrm{C} 28 & \mathrm{C} 29 & \mathrm{C} 30 \\ \text { Charge } & 0.355047 & 0.054106 & -0.220966 & -0.202133 & -0.140484 \\ \text { Atom } & \mathrm{C} 31 & \mathrm{C} 32 & \mathrm{C} 33 & \mathrm{C} 34 & \mathrm{C} 35 \\ \text { Charge } & 0.070289 & -0.219827 & -0.204139 & -0.132423 & 0.068701 \\ \text { Atom } & \mathrm{C} 36 & \mathrm{C} 37 & \mathrm{C} 38 & \mathrm{C} 39 & \mathrm{C} 40 \\ \text { Charge } & -0.218661 & -0.140054 & -0.202886 & -0.220664 & 0.077666 \\ \text { Atom } & \mathrm{C} 41 & \mathrm{C} 42 & \mathrm{H} 43 & \mathrm{H} 44 & \mathrm{H} 45 \\ \text { Charge } & -0.209862 & -0.137506 & 0.232048 & 0.233819 & 0.229272 \\ \text { Atom } & \mathrm{H} 46 & \mathrm{H} 47 & \mathrm{H} 48 & \mathrm{H} 49 & \mathrm{H} 50 \\ \text { Charge } & 0.231530 & 0.486314 & 0.497040 & 0.254205 & 0.253839 \\ \text { Atom } & \mathrm{H} 51 & \mathrm{H} 52 & \mathrm{H} 53 & \mathrm{H} 54 & \mathrm{H} 55 \\ \text { Charge } & 0.232146 & 0.246969 & 0.224937 & 0.202520 & 0.231262 \\ \text { Atom } & \mathrm{H} 56 & \mathrm{H} 57 & \mathrm{H} 58 & \mathrm{H} 59 & \mathrm{H} 60 \\ \text { Charge } & 0.247038 & 0.223320 & 0.195576 & 0.249296 & 0.235227 \\ \text { Atom } & \mathrm{H} 61 & \mathrm{H} 62 & \mathrm{H} 63 & \mathrm{H} 64 & \mathrm{H} 65 \\ \text { Charge } & 0.234497 & 0.245558 & 0.228584 & 0.214748 & 0.223058 \\ \text { Atom } & \mathrm{H} 66 & 067 & 068 & \mathrm{H} 69 & \mathrm{H} 70 \\ \text { Charge } & 0.234533 & -0.006686 & -0.851997 & 0.468291 & 0.452471\end{array}$

Sum of Mulliken atomic charges $=2.00000$

Transition state associated with oxygen releasing for complex $\mathbf{1}$ :

$\begin{array}{lccccc}\text { Atom } & \text { Mn1 } & \text { Mn2 } & 03 & 04 & 05 \\ \text { Spin } & -3.519410 & 3.627066 & -0.044015 & -0.027803 & 0.948050 \\ \text { Atom } & 06 & \mathrm{~N} 7 & \mathrm{~N} 8 & \mathrm{~N} 9 & \mathrm{~N} 10 \\ \text { Spin } & -0.004250 & -0.003987 & 0.030260 & -0.017686 & 0.034741 \\ \text { Atom } & \mathrm{N} 11 & \mathrm{~N} 12 & \mathrm{C} 13 & \mathrm{C} 14 & \mathrm{C} 15 \\ \text { Spin } & -0.017797 & 0.042636 & -0.012793 & -0.014897 & 0.009954 \\ \text { Atom } & \mathrm{C} 16 & \mathrm{C} 17 & \mathrm{C} 18 & \mathrm{C} 19 & \mathrm{C} 20 \\ \text { Spin } & -0.016495 & 0.008917 & -0.014640 & -0.001693 & 0.003706 \\ \text { Atom } & \mathrm{C} 21 & \mathrm{C} 22 & \mathrm{C} 23 & \mathrm{C} 24 & \mathrm{C} 25\end{array}$




\begin{tabular}{|c|c|c|c|c|c|}
\hline Spin & 0.010969 & -0.006849 & 0.012676 & -0.007135 & 0.011067 \\
\hline Atom & $\mathrm{C} 26$ & $\mathrm{C} 27$ & $\mathrm{C} 28$ & $\mathrm{C} 29$ & C30 \\
\hline Spin & 0.001044 & 0.002416 & 0.000507 & 0.001332 & 0.004968 \\
\hline Atom & C31 & C32 & C33 & C34 & C35 \\
\hline Spin & -0.001282 & 0.002379 & 0.002536 & 0.002186 & -0.013639 \\
\hline Atom & C36 & C37 & C38 & C39 & $\mathrm{C} 40$ \\
\hline Spin & 0.006539 & -0.017387 & 0.006774 & -0.000631 & -0.006651 \\
\hline Atom & C41 & C42 & H43 & $\mathrm{H} 44$ & H45 \\
\hline Spin & -0.000125 & -0.007506 & -0.000246 & -0.000434 & 0.000398 \\
\hline Atom & H46 & $\mathrm{H} 47$ & H48 & $\mathrm{H} 49$ & $\mathrm{H} 50$ \\
\hline Spin & 0.000322 & 0.001140 & 0.002145 & 0.000971 & -0.000759 \\
\hline Atom & H51 & H52 & H53 & H54 & H55 \\
\hline Spin & 0.000751 & -0.000402 & 0.000579 & 0.001502 & 0.000836 \\
\hline Atom & H56 & H57 & H58 & H59 & H60 \\
\hline Spin & -0.000239 & 0.000602 & 0.002601 & 0.001166 & -0.000929 \\
\hline Atom & $\mathrm{H} 61$ & $\mathrm{H} 62$ & $\mathrm{H} 63$ & $\mathrm{H} 64$ & H65 \\
\hline Spin & -0.000642 & 0.000544 & -0.000441 & -0.000667 & -0.000185 \\
\hline Atom & H66 & 067 & 068 & H69 & $\mathrm{H} 70$ \\
\hline Spin & -0.000117 & 0.975540 & 0.000997 & 0.000792 & 0.000123 \\
\hline
\end{tabular}




\section{References}

(1) Becke, A. D. Density-functional Thermochemistry. III. The Role of Exact Exchange. J. Chem. Phys. 1993, 98, 5648-5652.

(2) Lee, C.; Yang, W.; Parr, R. G. Development of the Colle-Salvetti correlation-energy formula into a functional of the electron density. Phys. Rev. B 1988, 37, 785-789.

(3) Zhao, Y.; Truhlar, D. G. A New Local Density Functional for Main-group Thermochemistry, Transition Metal Bonding, Thermochemical Kinetics, and Noncovalent Interactions. J. Chem. Phys. 2006, 125, 194101-194118.

(4) Zhao, Y.; Truhlar, D. G. Density Functionals with Broad Applicability in Chemistry. Acc. Chem. Res. 2008, 41,157-167.

(5) Zhao, Y.; Truhlar, D. G. The M06 Suite of Density Functionals for Main Group Thermochemistry, Thermochemical Kinetics, Noncovalent Interactions, Excited States, and Transition Elements: Two New Functionals and Systematic Testing of Four M06-class Functionals and 12 Other Functionals. Theor. Chem. Acc. 2008, 120, 215-241.

(6) Hay, P. J.; Wadt, W. R. Ab Initio Effective Core Potentials for Molecular Calculations. Potentials for K to Au Including the Outermost Core Orbitals. J. Chem. Phys. 1985, 82, 299-310.

(7) Mouesca, J. M. Density Functional Theory-broken Symmetry (DFT-BS) Methodology Applied to Electronic and Magnetic Properties of Bioinorganic Prosthetic Groups. J. Chem. Phys. 1981, 74, 5737-5743.

(8) Jeener, J.; Meier, B. H.; Bachmann, P.; Ernst, R. R. Investigation of Exchange Processes by Twodimensional NMR Spectroscopy. J. Chem. Phys. 1979, 71, 2546-2556.

(9) Thorn H.; Dunning J. Gaussian Basis Sets for Use in Correlated Molecular Calculations. I. The Atoms Boron through Neon and Hydrogen. J. Chem. Phys. 1989, 90, 1007-1023.

(10) Woon, D. E.; Dunning, T. H. Gaussian Basis Sets for Use in Correlated Molecular Calculations.

III. The atoms aluminum through argon. J. Chem. Phys. 1993, 98, 1358-1371.

(11) Tomasi, J.; Mennucci, B.; Cammi, R. Quantum Mechanical Continuum Solvation Models. Chem. Rev. 2005, 105, 2999-3093.

(12) Barone, V.; Cossi, M. Quantum Calculation of Molecular Energies and Energy Gradients in Solution by a Conductor Solvent Model. J. Phys. Chem. A 1998, 102, 1995-2001. 
(13) Cancès, M. T.; Mennucci, B.; Tomasi, J. A New Integral Equation Formalism for the Polarizable Continuum Model: Theoretical Background and Applications to Isotropic and Anisotropic Dielectrics. J. Chem.Phys. 1997, 107, 3032-3041.

(14) Mennucci, B.; Tomasi, J. Continuum Solvation Models: A New Approach to the Problem of Solute's Charge Distribution and Cavity Boundaries. J. Chem. Phys. 1997, 106, 5151-5158.

(15) Foresman, J. B.; Keith, T. A.; Wiberg, K. B.; Snoonian, J.; Frisch, M. J. Solvent Effects. 5. Influence of Cavity Shape, Truncation of Electrostatics, and Electron Correlation on ab Initio Reaction Field Calculations. J. Phys. Chem. 1996, 100, 16098-16104.

(16 Liptak, M. D.; Shields, G. C. Accurate pKa Calculations for Carboxylic Acids Using Complete Basis Set and Gaussian-n Models Combined with CPCM Continuum Solvation Methods. J. Am. Chem. Soc. 2001, 123, 7314-7319.

(17) Pliego, J. J. R.; Riveros, J. M. Gibbs Energy of Solvation of Organic Ions in Aqueous and Dimethyl Sulfoxide Solutions. Phys. Chem. Chem. Phys. 2002, 4, 1622-1627.

(18) Bahers, T. L.; Adamo C.; Ciofini, I. Theoretical Determination of the pKas of the 8Hydroxyquinoline-5-sulfonic acid: A DFT Based Approach. Chem. Phys. Lett. 2009, 472, 30-34.

(19) Klamt, A.; Schüürmann, G. COSMO: A New Approach to Dielectric Screening in Solvents with Explicit Expressions for the Screening Energy and its Gradient. J. Chem. Soc. Perkin Trans 1993, 2, 799-805.

(20) Mavros M. G.; Tsuchimochi T.; Kowalczyk T.; McIsaac A.; Wang L. P.; Voorhis T. V. What Can Density Functional Theory Tell Us about Artificial Catalytic Water Splitting? Inorg. Chem. 2014, 53, 6386-6397. 